\title{
Functional Calculus in Weighted Group Algebras
}

\author{
Jacek DZIUBANSKI ${ }^{\dagger}$, Jean LUDWIG $\ddagger \S$, \\ and Carine Molitor-Braun $\ddagger$
}

\author{
Institute of Mathematics \\ Wroclaw University \\ PI. Grunwaldzki 2/4 - Poland \\ jdziuban@math.uni.wroc.pl
}

\author{
Département de Mathématiques \\ Université de Metz \\ lle de Saulcy \\ F-57045 Metz cedex 1 - France \\ ludwig@poncelet.sciences . univ-metz.fr
}

\author{
Laboratoire de Mathématiques \\ Université du Luxembourg \\ 162A, Avenue de la Faïencerie \\ L-1511 Luxembourg - Luxembourg \\ carine.molitor@uni.lu
}

Recibido: 9 de Abril de 2003

Aceptado: 12 de Enero de 2004

\begin{abstract}
Let $G$ be a compactly generated, locally compact group with polynomial growth and let $\omega$ be a weight on $G$. We look for general conditions on the weight which allow us to develop a functional calculus on a total part of $L^{1}(G, \omega)$. This functional calculus is then used to study harmonic analysis properties of $L^{1}(G, \omega)$, such as the Wiener property and Domar's theorem.
\end{abstract}

Key words: weight, weighted group algebra, functional calculus, Wiener property

2000 Mathematics Subject Classification: 43A20

$\dagger^{\dagger}$ Supported by a Nato scholarship, Polish Grant from KBN, Foundation for Polish Science (Subsidy $3 / 99)$.

¥ Supported by the research grant CUL/01/014.

$\S$ Supported by the European Commission via Harmonic Analysis and Related Problems network. 


\section{Introduction}

Weighted group algebras occur in a lot of situations. In the abelian case, their harmonic analysis properties have been studied among others by Beurling ([1], [2]), Domar $([5])$, and Vretblad ([20]). Domar proves the following results: Let $G$ be a locally compact abelian group and $\omega$ a weight on $G$. For every neighbourhood $N$ of the identity 1 in $\hat{G}$ (the dual group of $G$ ), there exists $f_{N} \in L^{1}(G, \omega)$ such that $\operatorname{supp} \hat{f}_{N} \subset N$, $\hat{f}_{N}(\mathbf{1}) \neq 0$, if and only if the weight $\omega$ satisfies

$$
\sum_{n=1}^{+\infty} \frac{\ln \omega\left(x^{n}\right)}{n^{2}}<+\infty, \quad \forall x \in G .
$$

In case this is true, the algebra $L^{1}(G, \omega)$ has the Wiener property, i.e. for every proper closed ideal $I$ of $L^{1}(G, \omega)$, there exists $\chi \in \hat{G}$ such that

$$
I \subset\left\{f \in L^{1}(G, \omega) \mid \hat{f}(\chi)=0\right\} .
$$

For the group $G=\mathbf{R}$, Vretblad even proves a partial converse of the Wiener property. He shows the following: Let $\omega$ be a symmetric weight on $\mathbf{R}$ such that

$$
\int_{\mathbf{R}} \frac{\ln \omega(x)}{1+x^{2}} d x=\infty
$$

Assume moreover that $\omega(x)=\exp \left(\frac{\pi}{2}|x| q(x)\right)$ with $q$ decreasing on $\mathbf{R}_{+}$and $\omega$ increasing on $\mathbf{R}_{+}$. Then $L^{1}(\mathbf{R}, \omega)$ does not have the Wiener property. Let's write $n x$ instead of $x^{n}$, as the group $\mathbf{R}$ is additive. Notice that under the assumptions on $q$ and $\omega$,

$$
\sum_{n=1}^{+\infty} \frac{\ln \omega(n x)}{n^{2}}<+\infty \forall x \in \mathbf{R} \Longleftrightarrow \int_{\mathbf{R}} \frac{\ln \omega(x)}{1+x^{2}} d x<+\infty .
$$

Hence Vretblad shows that if $L^{1}(\mathbf{R}, \omega)$ has the Wiener property, then $\sum_{n=1}^{+\infty} \frac{\ln \omega(n x)}{n^{2}}<$ $+\infty$, for every $x \in \mathbf{R}$. The aim of the present paper consists in proving similar results for non abelian groups. Let $G$ be a locally compact group and $\hat{G}$ its dual, i.e. the space of equivalence classes of unitary topologically irreducible representations of $G$. The question about Domar's property is then the following: Let $N$ be an arbitrary open set in $\hat{G}$. Does there exist $f_{N}$ in the weighted group algebra $L^{1}(G, \omega)$ such that $\pi\left(f_{N}\right)=0$ for every $\pi \in \hat{G} \backslash N$ and $\rho\left(f_{N}\right) \neq 0$ for some given $\rho \in N$ ? The question about Wiener's property reads: If $I$ is a proper closed two-sided ideal in $L^{1}(G, \omega)$, does there exist $\pi \in \hat{G}$ such that $I \subset \operatorname{ker} \pi=\left\{f \in L^{1}(G, \omega) \mid \pi(f)=0\right\}$ ? The answer to both questions depends of course on the group $G$ and on the growth of the weight $\omega$.

Before stating our results, let us recall some precise definitions. A weight $\omega$ on a locally compact group $G$ is a Borel function that satisfies

$$
\omega: G \longrightarrow[1,+\infty[
$$


such that

$$
\omega(x y) \leq \omega(x) \omega(y) \quad \forall x, y \in G .
$$

All weights will be assumed to be symmetric in this paper, i.e.

$$
\omega\left(x^{-1}\right)=\omega(x) \quad \forall x \in G .
$$

Moreover we shall always require the function $\omega$ to be bounded on compact sets.

Recall also that the algebra $L^{1}(G, \omega)$ is then given by

$$
L^{1}(G, \omega)=\left\{f: G \rightarrow \mathbf{C} \mid f \text { measurable and }\|f\|_{\omega}=\int_{G}|f(x)||\omega(x)| d x<+\infty\right\} .
$$

Except for the question of the symmetry of the algebra $L^{1}(G, \omega)$, studied in [6], nothing seems to be known for non-abelian groups and for weights growing faster than sub-exponential ones. One may ask the question of what are the most general weights $\omega$ on a locally compact group $G$, such that the weighted group algebra $L^{1}(G, \omega)$ has reasonable harmonic analysis properties such as Domar's property or Wiener's property. The results known for the constant weight $\omega \equiv 1$ and the necessity to measure the growth of the weight urge us to limit ourselves to locally compact, compactly generated groups with polynomial growth. For such a group, let $U$ be a generating neighbourhood of the identity element $e$, with compact closure, i.e. such that

$$
G=\bigcup_{n=1}^{\infty} U^{n}
$$

and such that the Haar measure of $U$ satisfies

$$
\left|U^{n}\right| \leq K \cdot n^{Q}
$$

for some $K>0, Q \in \mathbf{N}$. One may define the following quantities:

$$
\tau_{U}(x)=|x|=\inf \left\{n \in \mathbf{N}^{*} \mid x \in U^{n}\right\}
$$

where $\mathbf{N}^{*}=\mathbf{N} \backslash\{0\}($ see 1.2$)$ and

$$
s(n)=\sup _{x \in U^{n}} \omega(x)
$$

If $G=\mathbf{R}, \tau_{U}(\cdot)=|\cdot|$ is equivalent to the absolute value in the following sense: If $U=[-1,1]$, then $\tau_{U}(x)-1<|x| \leq \tau_{U}(x)$ where $|x|$ denotes the absolute value of $x$. This justifies the use of the notation $|\cdot|$.

A weight $\omega$ is said to be sub-exponential of degree at most $\alpha, 0 \leq \alpha<1$, if there exists $C>0$ such that

$$
\omega(x) \leq e^{C|x|^{\alpha}}, \quad \forall x \in G
$$


(see 1.4). The way to prove the Wiener property is to use functional calculus to construct an approximate identity contained in the minimal ideal with empty hull, which implies, together with the symmetry of the algebra $L^{1}(G, \omega)$, the Wiener property. In [6] it is shown that if $\omega$ is sub-exponential, then $L^{1}(G, \omega)$ has Wiener's property. In this paper we prove the Wiener property even for faster growing weights, as well as other harmonic analysis properties of $L^{1}(G, \omega)$ (homeomorphism of $\operatorname{Prim}_{*} L^{1}(G)$ and $\operatorname{Prim}_{*} L^{1}(G, \omega)$, Domar's property, existence of minimal ideals of a given hull). We show that if the weight $\omega$ satisfies the condition

$$
\sum_{n \in \mathbf{N}, n \geq e^{e}} \frac{(\ln (\ln n)) \cdot \ln (s(n))}{n^{2}}<+\infty
$$

then $L^{1}(G, \omega)$ is symmetric and has Domar's and Wiener's properties. The condition (1) seems slightly stronger than Domar's condition. Nevertheless, for fast growing weights, the presence of the factor $(\ln (\ln n))$ does not seem to affect the result as the following example shows: Let $\omega$ be defined by

$$
\omega(x)=e^{C \frac{|x|}{(\ln (|x|+1)) \gamma}} .
$$

Condition (1) is satisfied for this weight if and only if $\gamma>1$. In this case, $L^{1}(G, \omega)$ has Domar's and Wiener's properties. On the other hand, if $G=\mathbf{R}$ and $\omega$ is as above, then the results of Domar and Vretblad show that $L^{1}(\mathbf{R}, \omega)$ has Domar's and Wiener's properties if and only if $\gamma>1$. This is the same condition on $\gamma$. Hence, for fast growing weights our result seems to be almost optimal, as we get the same condition on $\gamma$ as in the abelian case. Another weight studied in this paper is

$$
\omega(x)=e^{\sum_{k \in \mathrm{N}} c_{k}|x|^{\gamma_{k}}}
$$

where $0<\gamma_{k}<1, \gamma_{k} \uparrow 1, c_{k}>0, \sum_{k \in \mathbf{N}} c_{k}<+\infty$.

Our results are obtained thanks to the construction of a functional calculus on $L^{1}(G, \omega)$. In general, functional (or symbolic) calculus in function algebras is a special case of functional calculus in Banach $*$-algebras. Let's recall the following definition and facts: Let $\mathcal{B}$ be a Banach $*$-algebra. We say that a function $\varphi$ operates on an element $f$ of $\mathcal{B}$, if the Gelfand transform of $f$ with respect to the smallest commutative Banach subalgebra $\mathcal{A}$ of $\mathcal{B}$, containing $f$, is real and if there exists $g \in \mathcal{A}$ such that $\varphi \circ \hat{f}=\hat{g}$, where $\hat{f}$ and $\hat{g}$ denote the Gelfand transforms of $f$ and $g$ (see for instance [8], [10]). We then write $g=\varphi\{f\}$. The idea to realise this comes from the Fourier inversion theorem. Let $f$ be a self-adjoint element of $\mathcal{B}$ (if $\mathcal{B}$ is a symmetric *-algebra) and let's define

$$
u(n f)=\sum_{k=1}^{\infty} \frac{i^{k} n^{k}}{k !} f^{k}, \quad n \in \mathbf{Z} .
$$

Here $f^{k}$ is the $k$-th power of $f$ for the multiplication in the algebra $\mathcal{B}$. In case of $L^{1}(G)$ or $L^{1}(G, \omega)$ we write $f^{* k}$ to indicate that it is a convolution product. A 
periodic function $\varphi$ of period $2 \pi$ and such that $\varphi(0)=0$ then operates on $f$ through the formula

$$
\varphi\{f\}=\sum_{n \in \mathbf{Z}} u(n f) \hat{\varphi}(n)
$$

provided

$$
\sum_{n \in \mathbf{Z}}\|u(n f)\||\hat{\varphi}(n)|
$$

converges, where $\|\cdot\|$ denotes the norm in the Banach algebra $\mathcal{B}\left(\|\cdot\|=\|\cdot\|_{\omega}\right.$ in case of $\left.L^{1}(G, \omega)\right)$ and where $\hat{\varphi}$ is the Fourier transform of $\varphi$ on the interval $[0,2 \pi]$. Further details about the functional calculus for function algebras and its properties will be given in Section 2. Let's mention here among others the pioneer work of Dixmier (1960, [4]), Kahane (1970, [9]), Pytlik (1973 [17]; 1982, [18]) and Hulanicki (1974, [7]; 1984, [8]).

To prove the convergence of

$$
\sum_{n \in \mathbf{Z}} \| u(n f)|||\hat{\varphi}(n)|
$$

one needs of course a good bound for $\|u(n f)\|$ and a function $\varphi$ whose Fourier coefficients decrease rapidly enough. The existence of such functions $\varphi \not \equiv 0$ will depend on the growth of $\|u(n f)\|$, and hence on the weight $\omega$ (in case of a weighted group algebra). In fact, in order to have $\varphi \not \equiv 0$, the decrease of $|\hat{\varphi}(n)|$ must not be too rapid, especially as we also need some control on the support of the function $\varphi$ (in order to prove the desired harmonic analysis properties).

In [6] such a functional calculus was constructed for $L^{1}(G, \omega)$ if the weight $\omega$ is sub-exponential. This proof cannot be extended to faster growing weights. So a new approach to this problem will have to be developed in this paper. First the evaluation of the bound for $\|u(n f)\|_{\omega}\left(f=f^{*}\right.$ continuous with compact support) has to be improved considerably. This will be done in Section 3 by adapting and developing a method introduced by Hulanicki in [8]. Secondly, appropriate functions $\varphi$ that operate on $f$ have to be constructed. To do this we shall use a result of Paley-Wiener ([16]) and show that such functions exist if the growth of the weight $\omega$ is limited by the condition

$$
\sum_{n \in \mathbf{N}, n \geq e^{e}} \frac{(\ln (\ln n)) \ln (s(n))}{1+n^{2}}<+\infty,
$$

(see Section 4). Finally, there exist enough of such functions $\varphi$ to prove the harmonic analysis results we are interested in sections 5, 6 and 7 .

\section{Examples of weights}

1.1. In this chapter we recall some results of [6] and give some examples of weights. Part of these examples are already found in [6], but their presence in this paper is 
necessary for a better understanding of the abstract results that will follow.

1.2. Let $G$ be a compactly generated, locally compact group with polynomial growth. Let $U$ be an open, symmetric and relatively compact neighbourhood of the identity element $e$ of $G$ with $G=\bigcup_{n=1}^{+\infty} U^{n}$. Such a neighbourhood will be called a generating neighbourhood. As $G$ has polynomial growth, there exist constants $Q \in \mathbf{N}$, and $K>0$ such that the Haar measure of $U^{n}$ satisfies

$$
\left|U^{n}\right| \leq K n^{Q}
$$

Let $\tau_{U}: G \rightarrow[1,+\infty[$ be defined by

$$
\tau_{U}(x)=\inf \left\{n \in \mathbf{N}^{*} \mid x \in U^{n}\right\} .
$$

Then

$$
\tau_{U}(x \cdot y) \leq \tau_{U}(x)+\tau_{U}(y)
$$

It is easy to check that $\omega_{U}: G \rightarrow[1,+\infty[$ defined by

$$
\omega_{U}(x)=1+\tau_{U}(x)
$$

is a weight on $G$. The same is true for every $\omega_{U}(x)^{\alpha}, \alpha \in \mathbf{R}_{+}$(see [7], [8], [12]).

1.3 (Polynomial weights). A weight $\omega: G \rightarrow[1,+\infty[$ is said to be polynomial if and only if there exists $\alpha>0$ and $C>0$ such that

$$
\omega(x) \leq C\left(1+\tau_{U}(x)\right)^{\alpha}, \quad \forall x \in G .
$$

This definition is independent of the choice of the neighbourhood $U$. If $G$ is a connected, simply connected, nilpotent Lie group, this is equivalent to the fact that $\omega(x)$ is bounded by a polynomial function in the coordinates of the first or second kind of $x$.

1.4 (Sub-exponential weights). A weight $\omega: G \rightarrow[1,+\infty[$ is said to be subexponential of degree at most $\alpha, 0 \leq \alpha<1$, if there exists $C>0$ such that

$$
\omega(x) \leq e^{C\left(\tau_{U}(x)\right)^{\alpha}}, \quad \forall x \in G .
$$

This definition is independent of the choice of the neighbourhood $U$. Polynomial weights are sub-exponential and the function

$$
x \mapsto e^{C\left(\tau_{U}(x)\right)^{\alpha}},
$$

$0 \leq \alpha<1$, itself is a sub-exponential weight. 
1.5 (Condition $(S)$ ). (i) The condition $(S)$ on the weight $\omega$ is defined in order to get the symmetry of the algebra $L^{1}(G, \omega)$. A clue is given by what happens for $L^{1}(\mathbf{R}, \omega)$, where $\omega(x)=e^{\Phi(|x|)}$ with $\Phi: \mathbf{R}_{+} \rightarrow \mathbf{R}_{+}$. One knows that $L^{1}(\mathbf{R}, \omega)$ is symmetric if and only if $L^{1}(\mathbf{R}, \omega)$ admits no non-unitary characters (i.e. no characters $x \mapsto e^{c x}$ where $c \in \mathbf{C}$ has a non-zero real part), which is equivalent to the fact that

$$
\lim _{|x| \rightarrow+\infty} \frac{\ln \omega(x)}{|x|}=\lim _{|x| \rightarrow+\infty} \frac{\Phi(|x|)}{|x|}=0 .
$$

This condition is what Beurling ([1], [2]) and Vretblad ([20]) called the nonanalytic case.

(ii) Let now $G$ be an arbitrary compactly generated locally compact group with polynomial growth. Let $\omega$ be a weight on $G$ and $U$ a generating neighbourhood. If the weight $\omega$ is radial for $U$, i.e. if $\omega(x)=\omega\left(\tau_{U}(x)\right)=e^{\Phi\left(\tau_{U}(x)\right)}$ for some function $\Phi: \mathbf{N} \rightarrow \mathbf{R}_{+}$, then we may again define condition (S) by

$$
\lim _{\tau_{U}(x) \rightarrow+\infty} \frac{\Phi\left(\tau_{U}(x)\right)}{\tau_{U}(x)}=\lim _{k \rightarrow+\infty} \frac{\Phi(k)}{k}=0 .
$$

If the weight is not radial, we have to generalise the previous condition as follows.

(iii) Definition. For the given generating neighbourhood $U$, let us define

$$
s(0)=s_{0}=1 \quad \text { and } \quad s(k)=s_{k}=\sup _{x \in U^{k}} \omega(x) \quad \text { for } k \in \mathbf{N}^{*} .
$$

We say that the weight $\omega$ satisfies condition (S) if

$$
\lim _{k} \frac{\ln s_{k}}{k}=0 \quad \text { or, equivalently, } \quad \lim _{k} \sqrt[k]{s_{k}}=1 .
$$

(iv) As seen in [6], the condition $(S)$ is independent of the choice of the generating neighbourhood $U$.

(v) Assume that there exists a positive increasing function $\Phi: \mathbf{R}_{+} \rightarrow \mathbf{R}_{+}$with

$$
\lim _{k \rightarrow+\infty} \frac{\Phi(k)}{k}=0 \quad \text { and such that } \omega(x) \leq C \cdot e^{\Phi\left(\tau_{U}(x)\right)}, \quad \forall x \in G,
$$

for some positive constant $C$. Then $\omega$ satisfies condition $(S)$. This is in particular the case if $\omega$ is sub-exponential.

(vi) The function

$$
x \mapsto e^{\Phi\left(\tau_{U}(x)\right)}
$$

is itself a weight, if $\Phi$ is sub-additive. This is for instance the case if $\Phi$ is a positive, increasing, concave function. 
(vii) If $\omega$ satisfies $(S)$, then the algebra $L^{1}(G, \omega)$ is symmetric ([6]).

Example 1.6. Let $\omega(x)=e^{\sum_{n \geq 0} c_{n}\left(\tau_{U}(x)\right)^{\gamma_{n}}}$, with $0<\gamma_{n}<1, \gamma_{n} \uparrow 1, c_{n}>0$, $\sum_{n} c_{n}<+\infty$. Then $\Phi(x)=\sum_{n} c_{n} x^{\gamma_{n}}$ and $\omega$ satisfies condition $(S)$. The weight $\omega$ is not sub-exponential.

Examples 1.7. As previously, let $U$ be a generating neighbourhood. In order to simplify the notations, let us put $|x|=\tau_{U}(x)$ for every $x \in G$. Let $q: \mathbf{N} \rightarrow \mathbf{R}_{+}$be a decreasing function such that

$$
\lim _{n \rightarrow+\infty} q(n)=0 \quad \text { and } \quad \lim _{n \rightarrow+\infty}(n q(n))=+\infty .
$$

Then the function $\Phi: \mathbf{N} \rightarrow \mathbf{R}_{+}$defined by $\Phi(n)=n q(n)$ is sub-additive and $\omega: G \rightarrow$ $\mathbf{R}_{+}^{*}$ given by

$$
\omega(x)=e^{\Phi(|x|)}=e^{|x| q(|x|)}
$$

is a weight that satisfies condition $(S)$. Examples of such weights are for instance given by

(i) $\omega(x)=e^{C|x|^{\gamma}}=e^{|x| \frac{C}{|x|^{1-\gamma}}}, C>0,0<\gamma<1\left(|x|=\tau_{U}(x) \geq 1\right)$,

(ii) $\omega(x)=e^{\sum_{n \geq 0} c_{n} \frac{|x|}{1+|x| \beta_{n}}}=e^{|x| \sum_{n} \frac{c_{n}}{1+|x| \beta_{n}}}, 0<\beta_{n}<1,\left(\beta_{n}\right)_{n}$ decreasing, $\beta_{n} \rightarrow 0$, $\sum_{n} c_{n}<+\infty$

(iii) $\omega(x)=e^{C \frac{|x|}{\ln (e+|x|)}}$,

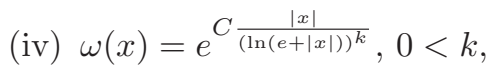

(v) $\omega(x)=e^{C \frac{|x|}{\ln (\ln (e+|x|))}}$.

The weights of examples (ii) to (v) are not sub-exponential. By $[6], L^{1}(G, \omega)$ is symmetric for each one of these weights.

Example 1.8. The weight $\omega(x)=e^{C|x|}$ does not satisfy condition (S). This implies that the algebra $L^{1}(G, \omega)$ is not symmetric $([6])$.

\section{Principles of functional calculus}

2.1. Let $G$ be a compactly generated, locally compact group with polynomial growth and let $\omega$ be a weight on $G$ satisfying a certain growth condition (to be specified later). The sub-exponential weights will be examples of such weights. One aim of this paper is the construction of a functional calculus for all self-adjoint functions $f=f^{*}$ that are continuous with compact support, where $f^{*}$ is defined by

$$
f^{*}(x)=\overline{f\left(x^{-1}\right)}, \quad \forall x \in G,
$$


the modular function of a group with polynomial growth being identically 1 . This construction will be inspired by the one given for $L^{1}(G)$ and $\mathcal{S}(G)$ in [4] and [8].

Let's recall the main ideas of this construction. It is based on the Fourier inversion formula: If $\varphi \in L^{1}([0,2 \pi])$ and if $\hat{\varphi}$ defined by

$$
\hat{\varphi}(n)=\frac{1}{2 \pi} \int_{0}^{2 \pi} \varphi(x) e^{-i n x} d x
$$

belongs to $l^{1}(\mathbf{Z})$, then

$$
\varphi(x)=\sum_{n \in \mathbf{Z}} e^{i n x} \hat{\varphi}(n) .
$$

The idea of functional calculus consists in replacing the variable $x$ by a function $f$ in the last formula. But as our function algebra $L^{1}(G, \omega)$ doesn't have a unit, $e^{\text {inf }}$ doesn't make sense in $L^{1}(G, \omega)$ and has to be replaced by

$$
u(n f)=\sum_{k=1}^{+\infty} \frac{i^{k}}{k !} n^{k} f^{* k}, \quad n \in \mathbf{Z} .
$$

We then look for non zero functions $\varphi: \mathbf{R} \rightarrow \mathbf{R}$, periodic with period $2 \pi$, such that

$$
\varphi\{f\}=\sum_{n \in \mathbf{Z}} u(n f) \hat{\varphi}(n)
$$

converges in $L^{1}(G, \omega)$. Because of the lack of a unit in $L^{1}(G, \omega)$ and because

$$
\sum_{n \in \mathbf{Z}} \hat{\varphi}(n)=\varphi(0),
$$

we have to restrict ourselves to functions $\varphi$ such that $\varphi(0)=0$. We also require the convergence of

$$
\sum_{n \in \mathbf{Z}}\|u(n f)\|_{\omega}|\hat{\varphi}(n)|
$$

Let us note that $u(n f) \in L^{1}(G, \omega)$ as

$$
\|u(n f)\|_{\omega} \leq e^{|n|\|f\|_{\omega}}<+\infty .
$$

But this bound will have to be improved quite a lot to allow the construction of functional calculus. This will be done in Section 3 and the results are stated in 3.12.

We then look for the non-zero functions $\varphi: \mathbf{R} \rightarrow \mathbf{R}$, periodic with period $2 \pi$, such that $\varphi(0)=0$ and such that (2) converges. Moreover, in order to establish harmonic analysis properties, we need to have a control on the support of the function $\varphi$. For instance, we want to be able to choose $\varphi$ such that $\operatorname{supp} \varphi \cap[0,2 \pi]$ is contained in a previously fixed compact set of $] 0,2 \pi[$. Of course a too strong decrease of the Fourier 
transform of $\varphi$ will then imply that this function will be identically zero, what we do not want. This will put some restrictions on the possible growth of $\|u(n f)\|_{\omega}$, and hence on the weight $\omega$. The functions $\varphi$ suitable for our purposes will be constructed in Section 4, thanks to a result of Paley-Wiener ([16]).

2.2. Functional calculus, if it exists (see Section 4), has the following properties:

For any continuous self-adjoint function $f=f^{*}$ with compact support, for any appropriate functions $\varphi$ and $\psi$,

$$
\left(\lambda_{1} \varphi+\lambda_{2} \psi\right)\{f\}=\lambda_{1} \varphi\{f\}+\lambda_{2} \psi\{f\}, \quad \forall \lambda_{1}, \lambda_{2} \in \mathbf{R} .
$$

If $\chi$ is any character of the abelian Banach $*$-algebra generated by $f$, then $\chi$ is continuous, $\chi(f)$ is real as $f$ is self-adjoint and

$$
\chi(\varphi\{f\})=\varphi(\chi(f)) .
$$

If $\pi$ is any $*$-representation of $L^{1}(G, \omega)$ on a Hilbert space $\mathcal{H}$, then

$$
\pi(\varphi\{f\})=\varphi(\pi(f))
$$

where $\varphi(\pi(f))$ is the operator obtained by the functional calculus of hermitian operators. then

If $\varphi$ and $\psi$ are functions such that $(\varphi \cdot \psi)^{\wedge}=\hat{\varphi} * \hat{\psi}$ still has appropriate decrease,

$$
(\varphi \cdot \psi)\{f\}=\varphi\{f\} * \psi\{f\} .
$$

To see this, one may apply the left regular representation, which is injective, to $(\varphi \cdot \psi)\{f\}$.

In particular, if $\varphi$ is such that $\operatorname{supp} \varphi \cap[0,2 \pi] \subset] 0,2 \pi[$ and such that $\hat{\varphi}$ has an appropriate decrease, then, there exists a function $\psi$ with the same properties and such that $\psi \equiv 1$ on $\operatorname{supp} \varphi$. Hence

$$
\varphi\{f\} * \psi\{f\}=\psi\{f\} * \varphi\{f\}=(\varphi \cdot \psi)\{f\}=\varphi\{f\} .
$$

Similar results are for instance already found in [4].

2.3. Instead of the "discrete" definition $\varphi\{f\}=\sum_{n \in \mathbf{Z}} u(n f) \hat{\varphi}(n)$, one may also use a "continuous" definition given by $\varphi\{f\}=\frac{1}{2 \pi} \int_{\mathbf{R}} u(\lambda f) \hat{\varphi}(\lambda) d \lambda$ for a real-valued $\mathcal{C}^{\infty}$-function $\varphi$ with compact support, such that $\varphi(0)=0$ and such that $\hat{\varphi}$ has an appropriate decrease. The properties of functional calculus remain of course the same. Moreover, one may also use results of Mandelbrojt ([13], [14]) to construct such functions $\varphi$. 


\section{Computation of the bound used in functional calculus}

3.1. Let $\omega$ be an arbitrary weight on $G$ (bounded on compact sets). Then there exists a constant $C>0$ such that

$$
\omega(x) \leq e^{C|x|}, \quad \forall x \in G,
$$

because every weight is exponentially bounded. For further purposes, let's fix this constant $C>1$, which is always possible. We define three other weights that will be useful in the computation of the bound. First, let's put

$$
\begin{aligned}
& \omega_{1}(x)=s(|x|)=\sup _{y \in U^{|x|}} \omega(y), \\
& \omega_{2}(x)=e^{C|x|},
\end{aligned}
$$

Let's also choose a constant $C^{\prime}>0$ such that

$$
(1+|x|)^{\frac{Q}{2}} \omega_{1}(x) \omega_{2}(x) \leq e^{C^{\prime}|x|} .
$$

This is possible because the left hand side is again a weight (bounded on compact sets). Let's define the weight

$$
\omega_{3}(x)=e^{C^{\prime}|x|}, \quad \forall x \in G
$$

and let's put

$$
\begin{aligned}
& s_{2}(n)=\sup _{x \in U^{n}} \omega_{2}(x)=e^{C n}, \\
& s_{3}(n)=\sup _{x \in U^{n}} \omega_{3}(x)=e^{C^{\prime} n}, \quad \forall n \in \mathbf{N}^{*} .
\end{aligned}
$$

Let

$$
r: \mathbf{N} \rightarrow \mathbf{N}
$$

be an arbitrary increasing function, that will only be specified later. For the rest of this section $f=f^{*}$ will be a self-adjoint continuous function with compact support. Finally, for any subset $A$ of $G$, let's note $A^{c}=G \backslash A$. We are now in the position to start the computation of the bound of $\|u(n f)\|_{\omega}$ for all $n \in \mathbf{Z}$. To do this we shall adapt the methods introduced by [8] to our situation.

3.2. Let us use the following decomposition of $u(f)$ :

$$
u(f)=g+k
$$

with $g=u(f) \cdot \chi_{U^{r(n)}}, k=u(f) \cdot \chi_{\left(U^{r(n)}\right)^{c}}$, where $\chi_{A}$ stands for the characteristic function of the set $A$. For further purposes we compute

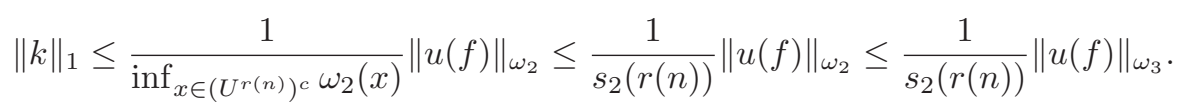


Similarly,

$$
\begin{aligned}
\|k\|_{\omega} & \leq \int_{G}|k(s)| \omega(s) d s \\
& \leq \int_{G}|k(s)|(1+|s|)^{\frac{Q}{2}} \omega_{1}(s) d s \\
& =\int_{\left(U^{r(n)}\right)^{c}}|k(s)|(1+|s|)^{\frac{Q}{2}} \omega_{1}(s) d s \\
& \leq \frac{1}{\inf _{x \in\left(U^{r(n)}\right)^{c}} \omega_{2}(x)} \int_{\left(U^{r(n)}\right)^{c}}|k(s)|(1+|s|)^{\frac{Q}{2}} \omega_{1}(s) \omega_{2}(s) d s \\
& \leq \frac{1}{s_{2}(r(n))}\|u(f)\|_{\omega_{3}} .
\end{aligned}
$$

3.3. Consider the unitary map $\left(u(f)+\delta_{e}\right): L^{2}(G) \longrightarrow L^{2}(G)$

$$
F \longmapsto\left(u(f)+\delta_{e}\right) * F=e^{i f} * F=u(f) * F+F,
$$

where $\delta_{e}$ is the Dirac measure. Obviously $\left\|\left(u(n f)+\delta_{e}\right) * F\right\|_{2}=\left\|e^{i n f} * F\right\|_{2} \leq\|F\|_{2}$ for $n \in \mathbf{Z}$. By 3.2,

$$
\begin{aligned}
\left\|\left(g+\delta_{e}\right) * F\right\|_{2} & =\left\|\left(u(f)+\delta_{e}-k\right) * F\right\|_{2} \\
& \leq\left(1+\|k\|_{1}\right)\|F\|_{2} \\
& \leq\left(1+\frac{\|u(f)\|_{\omega_{3}}}{s_{2}(r(n))}\right)\|F\|_{2}
\end{aligned}
$$

3.4. For $n \in \mathbf{N}$, let us make the following estimations (using the methods of [8]):

$$
\begin{aligned}
\left\|\left(\delta_{e}+u(f)\right)^{* n}\right\|_{\omega} & =\left\|\left(\left(\delta_{e}+g\right)+k\right)^{* n}\right\|_{\omega} \\
& \leq \sum_{m=0}^{n} \sum_{\mathbf{a}, \mathbf{b}}\left\|\left(\delta_{e}+g\right)^{a_{1}} * k^{b_{1}} * \cdots *\left(\delta_{e}+g\right)^{a_{n}} * k^{b_{n}}\right\|_{\omega},
\end{aligned}
$$

where $\mathbf{a}=\left(a_{1}, \ldots, a_{n}\right) \in\{0,1\}^{n}, \mathbf{b}=\left(b_{1}, \ldots, b_{n}\right) \in\{0,1\}^{n},|\mathbf{a}|=m,|\mathbf{b}|=n-m$, $a_{i}+b_{i}=1,\left(\delta_{e}+g\right)^{a_{j}}=\delta_{e}$ if $a_{j}=0$ and $k^{b_{j}}=\delta_{e}$ if $b_{j}=0$. Fix $\mathbf{a}$ and $\mathbf{b}$ as above. Assume that $\mathbf{a} \neq 0$. Then

$$
\begin{aligned}
&\left\|\left(\delta_{e}+g\right)^{a_{1}} * k^{b_{1}} * \cdots *\left(\delta_{e}+g\right)^{a_{n}} * k^{b_{n}}\right\|_{\omega} \\
& \leq \int_{G} \cdots \int_{G} \|\left(\delta_{e}+g\right)^{a_{1}} * \delta_{s_{1}}^{b_{1}} * \cdots *\left(\delta_{e}+g\right)^{a_{n}} * \delta_{s_{n}}^{b_{n}} \|_{\omega} \\
& \cdot\left|k\left(s_{1}\right)^{b_{1}} \cdots k\left(s_{n}\right)^{b_{n}}\right| d s_{1} \cdots d s_{n},
\end{aligned}
$$


where the convention is such that there is no integral with respect to $d s_{l}$ if $b_{l}=0$. One may check that

$$
\begin{aligned}
\left\|\left(\delta_{e}+g\right)^{a_{1}} * \delta_{s_{1}}^{b_{1}} * \cdots *\left(\delta_{e}+g\right)^{a_{n}} * \delta_{s_{n}}^{b_{n}}\right\|_{\omega} & \\
\leq \sum_{a_{j}=1}\left\|\left(\delta_{e}+g\right)^{a_{1}} * \delta_{s_{1}}^{b_{1}} * \cdots *\left(\delta_{e}+g\right)^{a_{j-1}} * \delta_{s_{j-1}}^{b_{j-1}} * g * \delta_{s_{j}}^{b_{j}} * \cdots * \delta_{s_{n}}^{b_{n}}\right\|_{\omega} & +\prod_{b_{i}=1} \omega\left(s_{i}\right) .
\end{aligned}
$$

Similarly,

$$
\begin{aligned}
\left\|e^{i n f} * F\right\|_{\omega} & =\left\|\left(\delta_{e}+u(f)\right)^{* n} * F\right\|_{\omega} \\
& \leq \sum_{m=0}^{n} \sum_{\mathbf{a}, \mathbf{b}}\left\|\left(\delta_{e}+g\right)^{a_{1}} * k^{b_{1}} * \cdots *\left(\delta_{e}+g\right)^{a_{n}} * k^{b_{n}} * F\right\|_{\omega}
\end{aligned}
$$

and

$$
\begin{aligned}
& \|\left(\delta_{e}+g\right)^{a_{1}} * k^{b_{1}} * \cdots *\left(\delta_{e}+g\right)^{a_{n}} * k^{b_{n}} * F \|_{\omega} \\
& \leq \int_{G} \cdots \int_{G}\left\|\left(\delta_{e}+g\right)^{a_{1}} * \delta_{s_{1}}^{b_{1}} * \cdots *\left(\delta_{e}+g\right)^{a_{n}} * \delta_{s_{n}}^{b_{n}} * F\right\|_{\omega} \\
& \cdot\left|k\left(s_{1}\right)^{b_{1}} \cdots k\left(s_{n}\right)^{b_{n}}\right| d s_{1} \cdots d s_{n} .
\end{aligned}
$$

Finally,

$$
\begin{aligned}
&\left\|\left(\delta_{e}+g\right)^{a_{1}} * \delta_{s_{1}}^{b_{1}} * \cdots *\left(\delta_{e}+g\right)^{a_{n}} * \delta_{s_{n}}^{b_{n}} * F\right\|_{\omega} \\
& \leq \sum_{a_{j}=1}\left\|\left(\delta_{e}+g\right)^{a_{1}} * \delta_{s_{1}}^{b_{1}} * \cdots *\left(\delta_{e}+g\right)^{a_{j-1}} * \delta_{s_{j-1}}^{b_{j-1}} * g * \delta_{s_{j}}^{b_{j}} * \cdots * \delta_{s_{n}}^{b_{n}} * F\right\|_{\omega} \\
&+\prod_{b_{i}=1} \omega\left(s_{i}\right)\|F\|_{\omega} .
\end{aligned}
$$

3.5. Let us denote $B(n)=U^{n}$. Then, for $\mathbf{a} \neq 0$, the function

$$
\left(\delta_{e}+g\right)^{a_{1}} * \delta_{s_{1}}^{b_{1}} * \cdots *\left(\delta_{e}+g\right)^{a_{j-1}} * \delta_{s_{j-1}}^{b_{j-1}} * g * \delta_{s_{j}}^{b_{j}} * \cdots * \delta_{s_{n}}^{b_{n}}
$$

is supported by $B\left(n r(n)+\left|s_{1}\right|^{b_{1}}+\cdots+\left|s_{n}\right|^{b_{n}}\right)$ with the convention that $\left|s_{l}\right|^{b_{l}}=0$ if $b_{l}=0$. Moreover, by 1.2 ,

$$
\left|B\left(n r(n)+\left|s_{1}\right|^{b_{1}}+\cdots+\left|s_{n}\right|^{b_{n}}\right)\right| \leq K \cdot(1+n r(n))^{Q} \prod_{b_{i}=1}\left(1+\left|s_{i}\right|\right)^{Q} .
$$

Similarly, the function $\left(\delta_{e}+g\right)^{a_{1}} * \delta_{s_{1}}^{b_{1}} * \cdots *\left(\delta_{e}+g\right)^{a_{j-1}} * \delta_{s_{j-1}}^{b_{j-1}} * g * \delta_{s_{j}}^{b_{j}} * \cdots * \delta_{s_{n}}^{b_{n}} * F$ is supported by $B\left(q+n r(n)+\left|s_{1}\right|^{b_{1}}+\cdots+\left|s_{n}\right|^{b_{n}}\right)$, if $\operatorname{supp} F \subset U^{q}$, and

$$
\left|B\left(q+n r(n)+\left|s_{1}\right|^{b_{1}}+\cdots+\left|s_{n}\right|^{b_{n}}\right)\right| \leq K \cdot(1+q)^{Q}(1+n r(n))^{Q} \prod_{b_{i}=1}\left(1+\left|s_{i}\right|\right)^{Q} .
$$


3.6. If $h$ is any function with compact support in $B(r)$, then

$$
\|h\|_{\omega}=\int_{U^{r}}|h(x)| \omega(x) d x \leq s(r)\|h\|_{2}|B(r)|^{\frac{1}{2}} .
$$

Obviously $\|g\|_{2} \leq\|u(f)\|_{2}$. Hence, by $3.3-3.5$, we obtain

$$
\begin{aligned}
& \left\|\left(\delta_{e}+g\right)^{a_{1}} * \delta_{s_{1}}^{b_{1}} * \cdots *\left(\delta_{e}+g\right)^{a_{n}} * \delta_{s_{n}}^{b_{n}}\right\|_{\omega} \\
& \leq \sum_{a_{j}=1}\left(1+\frac{\|u(f)\|_{\omega_{3}}}{s_{2}(r(n))}\right)^{a_{1}+\cdots+a_{j-1}} \cdot\|u(f)\|_{2} \cdot\left|B\left(n r(n)+\left|s_{1}\right|^{b_{1}}+\cdots+\left|s_{n}\right|^{b_{n}}\right)\right|^{\frac{1}{2}} \\
& \cdot s\left(n r(n)+\left|s_{1}\right|^{b_{1}}+\cdots+\left|s_{n}\right|^{b_{n}}\right)+\prod_{b_{i}=1} \omega\left(s_{i}\right) \\
& \leq K^{\frac{1}{2}}\|u(f)\|_{2} \sum_{a_{j}=1}\left(1+\frac{\|u(f)\|_{\omega_{3}}}{s_{2}(r(n))}\right)^{a_{1}+\cdots+a_{j-1}}(1+n r(n))^{\frac{Q}{2}} \\
& \cdot s\left(n r(n)+\left|s_{1}\right|^{b_{1}}+\cdots+\left|s_{n}\right|^{b_{n}}\right) \prod_{b_{i}=1}\left(1+\left|s_{i}\right|\right)^{\frac{Q}{2}}+\prod_{b_{i}=1} \omega\left(s_{i}\right) \\
& \leq K^{\frac{1}{2}}\|u(f)\|_{2} \cdot n(1+n r(n))^{\frac{Q}{2}} \cdot s(n r(n)) \cdot\left(1+\frac{\|u(f)\|_{\omega_{3}}}{s_{2}(r(n))}\right)^{n} \\
& \prod_{b_{i}=1}\left(\left(1+\left|s_{i}\right|\right)^{\frac{Q}{2}} \cdot \omega_{1}\left(s_{i}\right)\right)+\prod_{b_{i}=1} \omega\left(s_{i}\right) \\
& \leq K^{\frac{1}{2}}\|u(f)\|_{2} \cdot n(1+n r(n))^{\frac{Q}{2}} \cdot s(n r(n)) \cdot\left(\left(1+\frac{\|u(f)\|_{\omega_{3}}}{s_{2}(r(n))}\right)^{s_{2}(r(n))}\right)^{\frac{n}{s_{2}(r(n))}} \\
& \cdot \prod_{b_{i}=1}\left(\left(1+\left|s_{i}\right|\right)^{\frac{Q}{2}} \cdot \omega_{1}\left(s_{i}\right)\right)+\prod_{b_{i}=1} \omega\left(s_{i}\right) \\
& \leq K^{\frac{1}{2}}\|u(f)\|_{2} \cdot n(1+n r(n))^{\frac{Q}{2}} \cdot s(n r(n)) \cdot e^{\|u(f)\|_{\omega_{3}} \cdot\left(\frac{n}{s_{2}(r(n))}\right)} \\
& \cdot \prod_{b_{i}=1}\left(\left(1+\left|s_{i}\right|\right)^{\frac{Q}{2}} \cdot \omega_{1}\left(s_{i}\right)\right)+\prod_{b_{i}=1} \omega\left(s_{i}\right) \text {. }
\end{aligned}
$$

Similarly, if $\operatorname{supp} F \subset U^{q}$,

$$
\begin{aligned}
& \left\|\left(\delta_{e}+g\right)^{a_{1}} * \delta_{s_{1}}^{b_{1}} * \cdots *\left(\delta_{e}+g\right)^{a_{n}} * \delta_{s_{n}}^{b_{n}} * F\right\|_{\omega} \\
& \leq K^{\frac{1}{2}}\|u(f)\|_{1}\|F\|_{2}(1+q)^{\frac{Q}{2}} s(q) \cdot n(1+n r(n))^{\frac{Q}{2}} \cdot s(n r(n)) \cdot e^{\|u(f)\|_{\omega_{3}} \cdot\left(\frac{n}{s_{2}(r(n))}\right)} \\
& \cdot \prod_{b_{i}=1}\left(\left(1+\left|s_{i}\right|\right)^{\frac{Q}{2}} \cdot \omega_{1}\left(s_{i}\right)\right)+\|F\|_{\omega} \prod_{b_{i}=1} \omega\left(s_{i}\right)
\end{aligned}
$$


as $\left\|g * \delta_{s_{j}}^{b_{j}} * \cdots * \delta_{s_{n}}^{b_{n}} * F\right\|_{2} \leq\|g\|_{1}\|F\|_{2} \leq\|u(f)\|_{1}\|F\|_{2}$. Therefore for $\mathbf{a} \neq 0$ we have

$$
\begin{aligned}
& \left\|\left(\delta_{e}+g\right)^{a_{1}} * k^{b_{1}} * \cdots *\left(\delta_{e}+g\right)^{a_{n}} * k^{b_{n}}\right\|_{\omega} \\
& \leq K^{\frac{1}{2}}\|u(f)\|_{2} \cdot n(1+n r(n))^{\frac{Q}{2}} \cdot s(n r(n)) \cdot e^{\|u(f)\|_{\omega_{3}} \cdot\left(\frac{n}{s_{2}(r(n))}\right)} \\
& \cdot \prod_{b_{i}=1}\left(\int_{G}\left|k\left(s_{i}\right)\right|\left(1+\left|s_{i}\right|\right)^{\frac{Q}{2}} \omega_{1}\left(s_{i}\right) d s_{i}\right)+\prod_{b_{i}=1} \int_{G}\left|k\left(s_{i}\right)\right| \omega\left(s_{i}\right) d s_{i} \\
& \leq K^{\frac{1}{2}}\|u(f)\|_{2} \cdot n(1+n r(n))^{\frac{Q}{2}} \cdot s(n r(n)) \cdot e^{\|u(f)\|_{\omega_{3}} \cdot\left(\frac{n}{s_{2}(r(n))}\right)} \\
& \cdot \prod_{b_{i}=1}\left(\int_{G}\left|k\left(s_{i}\right)\right|\left(1+\left|s_{i}\right|\right)^{\frac{Q}{2}} \omega_{1}\left(s_{i}\right) d s_{i}\right)+\left(\frac{\|u(f)\|_{\omega_{3}}}{s_{2}(r(n))}\right)^{b_{1}+\cdots+b_{n}} .
\end{aligned}
$$

Similarly,

$$
\begin{aligned}
& \left\|\left(\delta_{e}+g\right)^{a_{1}} * k^{b_{1}} * \cdots *\left(\delta_{e}+g\right)^{a_{n}} * k^{b_{n}} * F\right\|_{\omega} \\
& \leq K^{\frac{1}{2}}\|u(f)\|_{1} \cdot(1+q)^{\frac{Q}{2}} s(q)\|F\|_{2} \cdot n(1+n r(n))^{\frac{Q}{2}} \cdot s(n r(n)) \cdot e^{\|u(f)\|_{\omega_{3}} \cdot\left(\frac{n}{s_{2}(r(n))}\right)} \\
& \cdot \prod_{b_{i}=1}\left(\int_{G}\left|k\left(s_{i}\right)\right|\left(1+\left|s_{i}\right|\right)^{\frac{Q}{2}} \omega_{1}\left(s_{i}\right) d s_{i}\right)+\|F\|_{\omega} \prod_{b_{i}=1} \int_{G}\left|k\left(s_{i}\right)\right| \omega\left(s_{i}\right) d s_{i} \\
& \leq K^{\frac{1}{2}}\|u(f)\|_{1} \cdot(1+q)^{\frac{Q}{2}} s(q)\|F\|_{2} \cdot n(1+n r(n))^{\frac{Q}{2}} \cdot s(n r(n)) \cdot e^{\|u(f)\|_{\omega_{3}} \cdot\left(\frac{n}{s_{2}(r(n))}\right)} \\
& \cdot \prod_{b_{i}=1}\left(\int_{G}\left|k\left(s_{i}\right)\right|\left(1+\left|s_{i}\right|\right)^{\frac{Q}{2}} \omega_{1}\left(s_{i}\right) d s_{i}\right)+\|F\|_{\omega}\left(\frac{\|u(f)\|_{\omega_{3}}}{s_{2}(r(n))}\right)^{b_{1}+\cdots+b_{n}}
\end{aligned}
$$

3.7. As

$$
\begin{aligned}
\int_{G}\left|k\left(s_{i}\right)\right|\left(1+\left|s_{i}\right|\right)^{\frac{Q}{2}} \omega_{1}\left(s_{i}\right) d s_{i} & =\int_{\left(U^{r(n)}\right)^{c}}\left|k\left(s_{i}\right)\right|\left(1+\left|s_{i}\right|\right)^{\frac{Q}{2}} \omega_{1}\left(s_{i}\right) d s_{i} \\
& \leq \frac{1}{s_{2}(r(n))} \int_{\left(U^{r(n)}\right) c}\left|k\left(s_{i}\right)\right|\left(1+\left|s_{i}\right|\right)^{\frac{Q}{2}} \omega_{1}\left(s_{i}\right) \omega_{2}\left(s_{i}\right) d s_{i} \\
& \leq \frac{1}{s_{2}(r(n))}\|u(f)\|_{\omega_{3}},
\end{aligned}
$$

we have by 3.2 and 3.6 , for $\mathbf{a} \neq 0$,

$$
\begin{aligned}
& \left\|\left(\delta_{e}+g\right)^{a_{1}} * k^{b_{1}} * \cdots *\left(\delta_{e}+g\right)^{a_{n}} * k^{b_{n}}\right\|_{\omega} \\
& \leq\left(K^{\frac{1}{2}}\|u(f)\|_{2} \cdot n(1+n r(n))^{\frac{Q}{2}} s(n r(n)) \cdot e^{\|u(f)\|_{\omega_{3}} \cdot\left(\frac{n}{s_{2}(r(n))}\right)}+1\right) \\
& \cdot\left(\frac{\|u(f)\|_{\omega_{3}}}{s_{2}(r(n))}\right)^{b_{1}+\cdots+b_{n}} \\
& \leq C_{1}(1+n)(1+n r(n))^{\frac{Q}{2}} s(n r(n)) \cdot e^{\|u(f)\|_{\omega_{3}} \cdot\left(\frac{n}{s_{2}(r(n))}\right)} \\
& \cdot\left(\frac{\|u(f)\|_{\omega_{3}}}{s_{2}(r(n))}\right)^{b_{1}+\cdots+b_{n}},
\end{aligned}
$$


where $C_{1}=\left(K^{\frac{1}{2}}\|u(f)\|_{2}+1\right)$. Analogously, for $F$ supported by $U^{q}$, we have

$$
\begin{aligned}
& \left\|\left(\delta_{e}+g\right)^{a_{1}} * k^{b_{1}} * \cdots *\left(\delta_{e}+g\right)^{a_{n}} * k^{b_{n}} * F\right\|_{\omega} \\
& \quad \leq C_{2}(1+n)(1+n r(n))^{\frac{Q}{2}} s(n r(n)) \cdot e^{\|u(f)\|_{\omega_{3}} \cdot\left(\frac{n}{s_{2}(r(n))}\right)} \cdot\left(\frac{\|u(f)\|_{\omega_{3}}}{s_{2}(r(n))}\right)^{b_{1}+\cdots+b_{n}},
\end{aligned}
$$

with $C_{2}=K^{\frac{1}{2}}\|u(f)\|_{1} \cdot(1+q)^{\frac{Q}{2}} s(q)\|F\|_{2}+\|F\|_{\omega}$.

3.8. For $\mathbf{a}=0$, we have $b_{1}+\cdots+b_{n}=n$ and

$$
\left\|\left(\delta_{e}+g\right)^{a_{1}} * k^{b_{1}} * \cdots *\left(\delta_{e}+g\right)^{a_{n}} * k^{b_{n}}\right\|_{\omega}=\left\|k^{* n}\right\|_{\omega} \leq\|k\|_{\omega}^{n} \leq\left(\frac{\|u(f)\|_{\omega_{3}}}{s_{2}(r(n))}\right)^{n} .
$$

Similarly

$$
\begin{aligned}
\left\|\left(\delta_{e}+g\right)^{a_{1}} * k^{b_{1}} * \cdots *\left(\delta_{e}+g\right)^{a_{n}} * k^{b_{n}} * F\right\|_{\omega} & =\left\|k^{* n} * F\right\|_{\omega} \\
& \leq\|F\|_{\omega}\|k\|_{\omega}^{n} \leq\|F\|_{\omega}\left(\frac{\|u(f)\|_{\omega_{3}}}{s_{2}(r(n))}\right)^{n} .
\end{aligned}
$$

This shows that the bounds of 3.7 remain valid even for $\mathbf{a}=0$ with the same constants $C_{1}, C_{2}$.

3.9. Using $3.4,3.7$, and 3.8 , we get the following bounds:

$$
\begin{aligned}
& \left\|\left(\delta_{e}+u(f)\right)^{* n}\right\|_{\omega} \\
& \leq C_{1}(1+n)(1+n r(n))^{\frac{Q}{2}} s(n r(n)) \cdot e^{\|u(f)\|_{\omega_{3}} \cdot\left(\frac{n}{s_{2}(r(n))}\right)} \\
& \cdot \sum_{m=0}^{n} \sum_{\mathbf{a}, \mathbf{b}} 1 \cdot\left(\frac{\|u(f)\|_{\omega_{3}}}{s_{2}(r(n))}\right)^{b_{1}+\cdots+b_{n}} \\
& =C_{1}(1+n)(1+n r(n))^{\frac{Q}{2}} s(n r(n)) \cdot e^{\|u(f)\|_{\omega_{3}} \cdot\left(\frac{n}{s_{2}(r(n))}\right)} \\
& \cdot\left(1+\frac{\|u(f)\|_{\omega_{3}}}{s_{2}(r(n))}\right)^{n} \\
& \leq C_{1}(1+n)(1+n r(n))^{\frac{Q}{2}} s(n r(n)) \cdot e^{2\|u(f)\|_{\omega_{3}} \cdot\left(\frac{n}{s_{2}(r(n))}\right)} .
\end{aligned}
$$

Likely,

$$
\begin{aligned}
\left\|\left(\delta_{e}+u(f)\right)^{* n} * F\right\|_{\omega} & =\left\|e^{i n f} * F\right\|_{\omega} \\
& \leq C_{2}(1+n)(1+n r(n))^{\frac{Q}{2}} s(n r(n)) \cdot e^{2\|u(f)\|_{\omega_{3}} \cdot\left(\frac{n}{s_{2}(r(n))}\right)} .
\end{aligned}
$$

The constants $C_{1}, C_{2}$ are the same as in 3.8. Finally,

$$
\begin{aligned}
\|u(n f)\|_{\omega} & \leq\left\|\left(\delta_{e}+u(f)\right)^{* n}\right\|_{\omega}+\omega(e) \\
& \leq\left(C_{1}+\omega(e)\right)(1+n)(1+n r(n))^{\frac{Q}{2}} s(n r(n)) \cdot e^{2\|u(f)\|_{\omega_{3}} \cdot\left(\frac{n}{s_{2}(r(n))}\right)} .
\end{aligned}
$$


3.10. The previous bounds have been proven for $n \in \mathbf{N}$. If we want a bound valid for $n \in \mathbf{Z}$, we first put $n f=(-n)(-f)$ if $n<0$. We replace $\|u(f)\|_{2}$ by $\max \left(\|u(f)\|_{2},\|u(-f)\|_{2}\right)$ in the constant $C_{1}$ and $\|u(f)\|_{1}$ by $\max \left(\|u(f)\|_{1},\|u(-f)\|_{1}\right)$ in the constant $C_{2}$. We then have

$$
\|u(n f)\|_{\omega} \leq C_{1}^{\prime}(1+|n|)(1+|n| r(|n|))^{\frac{Q}{2}} s(|n| r(|n|)) \cdot e^{C_{3}^{\prime}\left(\frac{|n|}{s_{2}(r(|n|))}\right)}, \quad n \in \mathbf{Z},
$$

and

$$
\left\|e^{i n f} * F\right\|_{\omega} \leq C_{2}^{\prime}(1+|n|)(1+|n| r(|n|))^{\frac{Q}{2}} s(|n| r(|n|)) \cdot e^{C_{3}^{\prime}\left(\frac{|n|}{\left.s_{2}(r|n|)\right)}\right)}, \quad n \in \mathbf{Z},
$$

where the constants $C_{1}^{\prime}, C_{2}^{\prime}, C_{3}^{\prime}$ are obtained in the following way:

$$
\begin{aligned}
C_{1}^{\prime} & =K^{\frac{1}{2}} e^{\|f\|_{2}}+1+\omega(e) \\
& \geq K^{\frac{1}{2}} \max \left(\|u(f)\|_{2},\|u(-f)\|_{2}\right)+1+\omega(e) \\
C_{2}^{\prime} & =K^{\frac{1}{2}} e^{\|f\|_{1}}(1+q)^{\frac{Q}{2}} s(q)\|F\|_{2}+\|F\|_{\omega} \\
& \geq K^{\frac{1}{2}} \max \left(\|u(f)\|_{1},\|u(-f)\|_{1}\right)(1+q)^{\frac{Q}{2}} s(q)\|F\|_{2}+\|F\|_{\omega},
\end{aligned}
$$

where $q \in \mathbf{N}^{*}$ is such that $\operatorname{supp} F \subset U^{q}$. As $f$ has compact support, there exists $p \in \mathbf{N}^{*}$ such that $\operatorname{supp} f \subset U^{p}$. Hence

$$
\|f\|_{\omega_{3}} \leq e^{C^{\prime} p}\|f\|_{1} \leq e^{C^{\prime} p}\|f\|_{\omega}
$$

and

$$
C_{3}^{\prime}=2 e^{\left(e^{C^{\prime} p}\right)\|f\|_{\omega}} \geq 2 e^{\|f\|_{\omega_{3}}} \geq 2 \max \left(\|u(f)\|_{\omega_{3}},\|u(-f)\|_{\omega_{3}}\right) .
$$

So the constants depend essentially only on the original weight $\omega$ and on the functions $f$ and $F$.

3.11 (Choice of the radius $r(n)$ ). Motivated by the techniques of functional calculus exposed later on in this paper, let's put

$$
r(n)=[\ln (\ln n)+1], \quad \forall n \geq e^{e},
$$

where $[z]$ denotes the integer part of $z$. Then

$$
\ln (\ln |n|) \leq r(|n|) \leq \ln (\ln |n|)+1 \leq 2 \ln (\ln |n|), \quad \text { for }|n| \geq e^{e}
$$

and

$$
s_{2}(r(|n|)) \geq e^{C(\ln (\ln |n|))}=(\ln |n|)^{C} .
$$

Hence

$$
e^{C_{3}^{\prime} \frac{|n|}{s_{2}(r(n \mid))}} \leq e^{C_{3}^{\prime} \frac{|n|}{(\ln |n|)^{C}}}
$$

and

$$
s(|n| r(|n|)) \leq s(|n|)^{r(|n|)} \leq s(|n|)^{2 \ln (\ln |n|)}, \quad \text { for }|n| \geq e^{e} .
$$

With this choice of the radius we get the following bounds: 
Theorem 3.12. Let $G$ be a locally compact, compactly generated group with polynomial growth. Let $\omega$ be an arbitrary weight on $G$. Let $f=f^{*}: G \rightarrow \mathbf{C}$ be continuous with compact support. Let $F: G \rightarrow \mathbf{C}$ be an arbitrary continuous function with compact support. We have the following bounds: There exist strictly positive constants $K_{1}, K_{2}, K_{3}$ such that

$$
\begin{gathered}
\|u(n f)\|_{\omega} \leq K_{1}(1+|n|)\left(1+|n|^{2}\right)^{\frac{Q}{2}} s(|n|)^{2 \ln (\ln |n|)} e^{K_{3}\left(\frac{|n|}{(\ln |n|)^{C}}\right)} \\
\left\|e^{i n f} * F\right\|_{\omega} \leq K_{2}(1+|n|)\left(1+|n|^{2}\right)^{\frac{Q}{2}} s(|n|)^{2 \ln (\ln |n|)} e^{K_{3}\left(\frac{|n|}{(\ln |n|)^{C}}\right)},
\end{gathered}
$$

for $|n| \geq e^{e}$. If $U$ is a generating neighbourhood of $G$ and $p, q \in \mathbf{N}^{*}$ such that supp $f \subset U^{p}$ and $\operatorname{supp} F \subset U^{q}$, then

$$
\begin{aligned}
& K_{1}=2^{\frac{Q}{2}}\left(K^{\frac{1}{2}} e^{\|f\|_{2}}+1+\omega(e)\right) \\
& K_{2}=2^{\frac{Q}{2}}\left(K^{\frac{1}{2}} e^{\|f\|_{1}}(1+q)^{\frac{Q}{2}} s(q)\|F\|_{2}+\|F\|_{\omega}\right) \\
& K_{3}=2 e^{\left(e^{C^{\prime} p}\right)\|f\|_{\omega}}
\end{aligned}
$$

where the constants $C, K$ and $C^{\prime}$ just depend on the growth of the weight $\omega$ and where $C>1$.

Proof. By 3.10 and 3.11 .

\section{Functional calculus}

4.1. In this section we shall first construct functions $\varphi$ whose Fourier transform have a strong decrease and may hence operate, under certain conditions, on the self-adjoint functions of $\mathcal{C}_{c}^{\infty}$. This will be done using a result of Paley-Wiener. The use of these functions in connection with the bound of $\|u(n f)\|_{\omega}$ obtained in 3.10, will give us a condition on the growth of the weight $\omega$. This condition will ensure the existence of functional calculus on a total part of $L^{1}(G, \omega)$ and will be called the non-abelian Beurling-Domar condition, because of its similarity with the results of Beurling and Domar.

4.2 (A result of Paley-Wiener). For any function $f \in L^{2}(\mathbf{R})$ the following two propositions are equivalent:

(i) There is a function $g \in L^{2}(\mathbf{R})$ such that $|g|=|f|$ and $\hat{g}$ vanishes on a half line.

(ii) $\int_{\mathbf{R}} \frac{|\ln | f(x)||}{1+x^{2}} d x<+\infty$.

See [16] or [19]. We shall apply the previous result in the following situation:

4.3. (a) Let $t: \mathbf{R} \rightarrow \mathbf{R}$ be such that 
(i) $t \geq 1$,

(ii) $t(-x)=t(x), \forall x \in \mathbf{R}$,

(iii) $t(x+y) \leq C t^{k}(x) t^{k}(y)$ for some $k \geq 1, C>0, \forall x, y \in \mathbf{R}$,

(iv) $\int_{\mathbf{R}} \frac{\ln (t(x))}{1+x^{2}} d x<+\infty$

If moreover, there exists $l \in \mathbf{N}$ such that

(v) $t^{-l} \in L^{2}(\mathbf{R})$,

(vi) $t^{-(l-k)} \in L^{1}(\mathbf{R})$,

then there is a function $g \in L^{1}\left(\mathbf{R}, t^{k}\right)$ such that supp $\hat{g} \in[0,+\infty[$.

In fact, this results from Paley-Wiener if we take $f=t^{-l}$.

(b) By translating the function $\hat{g}$ we may construct a function $g_{1}$ such that $\left|g_{1}\right|=$ $|g|=|f|$ and $\operatorname{supp} \hat{g}_{1} \subset[-\varepsilon,+\infty[$ for any given $\varepsilon>0$.

By taking $g_{2}=\check{g_{1}}$, where $\check{g}_{1}(x)=g_{1}(-x)$, we get a function $g_{2} \in L^{1}\left(\mathbf{R}, t^{k}\right)$ such that $\left.\left.\operatorname{supp} \hat{g}_{2} \subset\right]-\infty, \varepsilon\right]$.

Finally the function $h=g_{1} * g_{2}$ is in $L^{1}(\mathbf{R}, t)$ as $g_{1}, g_{2} \in L^{1}\left(\mathbf{R}, t^{k}\right)$ and as $t(x+y) \leq C t^{k}(x) t^{k}(y)$. Moreover supp $\hat{h} \subset[-\varepsilon, \varepsilon]$.

(c) Let now $\varphi_{1}$ be the inverse Fourier transform of $h$. Then $\varphi_{1} \in \mathcal{C}_{c}(\mathbf{R})$ such that $\operatorname{supp} \varphi_{1} \subset[-\varepsilon, \varepsilon]$ and such that $\hat{\varphi}_{1}=h \in L^{1}(\mathbf{R}, t)$. If, moreover, $\left|x^{r}\right| \leq K_{r} t(x)$ for all $x \in \mathbf{R}$, for all $r \in \mathbf{N}$, for some constant $K_{r}$ (depending on $r$ ), then $\hat{\varphi}_{1} \in L^{1}\left(\mathbf{R},\left|x^{r}\right|\right)$ for all $r \in \mathbf{N}$ and $\varphi_{1} \in \mathcal{C}_{c}^{\infty}(\mathbf{R})$. Moreover, translating the function $\varphi_{1}$ doesn't change the growth of its Fourier transform, so the Fourier transform of the translated function stays in $L^{1}(\mathbf{R}, t)$.

(d) Assume now that $\varphi_{1} \in \mathcal{C}_{c}(\mathbf{R})$ such that $\hat{\varphi}_{1} \in L^{1}(\mathbf{R}, t)$ and $\operatorname{supp} \varphi_{1} \subset[c, d]$. Let $I=[a, b]$ and consider $\varphi=\varphi_{1} * \chi_{I}$ where $\chi_{I}$ is the characteristic function of the interval $I$. It is easy to check that $\operatorname{supp} \varphi \subset[a+c, b+d]$ and that $\varphi \equiv 1$ on $[a+d, c+b]$ (provided $a+d<c+b$ ). Moreover,

$$
|\hat{\varphi}(\lambda)| \leq\left\|\hat{\chi}_{I}\right\|_{\infty} \cdot\left|\hat{\varphi}_{1}(\lambda)\right| \leq \frac{b-a}{2 \pi}\left|\hat{\varphi}_{1}(\lambda)\right|
$$

and $\hat{\varphi} \in L^{1}(\mathbf{R}, t)$. As a matter of fact,

$$
\begin{aligned}
|\hat{\varphi}(\lambda)| & \leq \frac{b-a}{2 \pi}\left|\hat{\varphi}_{1}(\lambda)\right| \\
& =\frac{b-a}{2 \pi}\left|g_{1} * g_{2}(\lambda)\right| \\
& \leq \frac{b-a}{2 \pi}|g| *|\check{g}|(\lambda) .
\end{aligned}
$$


Let now be two intervals $[q, r] \subset[q-\varepsilon, r+\varepsilon]$. It is then possible to construct a continuous function $\varphi, \operatorname{supp} \varphi \subset[q-\varepsilon, r+\varepsilon], \varphi \equiv 1$ on $[q, r]$ and $\hat{\varphi}(\lambda) \in L^{1}(\mathbf{R}, t)$. We just have to check that it is possible to find $a, b, c, d, a<b, c<d$, $a+d<c+b$, such that

$$
\left\{\begin{array}{l}
a+d=q, \\
c+b=r, \\
a+c=q-\varepsilon, \\
b+d=r+\varepsilon .
\end{array}\right.
$$

As a matter of fact, solving this system gives us $c=k, b=r-k, d=\varepsilon+k$, $a=q-\varepsilon-k$ with $k \in \mathbf{R}$ arbitrary. If $\left|x^{r}\right| \leq K_{r} t(x)$ for all $x \in \mathbf{R}$, for all $r \in \mathbf{N}$, for constants $K_{r}$, then $\varphi \in \mathcal{C}_{c}^{\infty}(\mathbf{R})$.

Next we shall show that there are similar results for $\mathbf{R} / 2 \pi \mathbf{Z}$, resp. $\mathbf{Z}$.

4.4. Let $w: \mathbf{Z} \rightarrow \mathbf{Z}$ be such that

(i) $w \geq 1$, increasing on $\mathbf{N}$,

(ii) $w(-n)=w(n) \quad \forall n \in \mathbf{Z}$,

(iii) $w(n+m) \leq C w^{k}(n) w^{k}(m)$ for some $k \geq 1, C \geq 1, \forall n, m \in \mathbf{N}$,

(iv) $\sum_{n \in \mathbf{N}} \frac{\ln w(n)}{1+n^{2}}<+\infty$.

Assume moreover that there exists $l_{1} \in \mathbf{N}$ such that

(v) $\sum_{n \in \mathbf{N}} w^{-2 l_{1} k}(n)<+\infty$,

(vi) $\sum_{n \in \mathbf{N}} w^{-\left(l_{1}-k^{2}\right)}(n)<+\infty$.

This has the following consequences for the function $w$ :

(a) First of all one has

$$
w(n+m) \leq C w^{k}(n) w^{k}(m) \quad \forall n, m \in \mathbf{Z} .
$$

For $n, m \in \mathbf{Z}_{-}$this follows from the symmetry of the function $w$. If $n \geq 0$, $m \leq 0, n+m \geq 0$, then

$$
w(n+m) \leq w(n) \leq C w^{k}(n) \leq C w^{k}(n) w^{k}(m)
$$

as $C \geq 1, w$ increasing on $\mathbf{N}$ and $k \geq 1$. The other cases are treated similarly.

(b) As $k \leq k^{2}$, one also has

$$
\sum_{n \in \mathbf{N}} w^{-\left(l_{1}-k\right)}(n)<+\infty .
$$


(c) If we put $l=l_{1} \cdot k$, then $l_{1} \leq l$ and

$$
\sum_{n \in \mathbf{N}} w^{-\left(l-k^{2}\right)}(n)<+\infty .
$$

(d) Thanks to the symmetry of the function $w$, all the sums in this section may be taken over $\mathbf{Z}$ instead of $\mathbf{N}$.

We may now prove the following result:

Proposition 4.5. Let $w$ be as in 4.4. Then, given $p, q, \varepsilon$ such that $0<p<p+\varepsilon<$ $q-\varepsilon<q<2 \pi$, there exists $\psi \in \mathcal{C}_{c}(\mathbf{R} / 2 \pi \mathbf{Z})$ such that

(i) $\operatorname{supp} \psi \cap[0,2 \pi] \subset[p, q]$,

(ii) $\psi \equiv 1$ on $[p+\varepsilon, q-\varepsilon]$,

(iii) $\sum_{n \in \mathbf{Z}}|\hat{\psi}(n)| w(n)<+\infty$,

i.e. $\hat{\psi} \in l^{1}(\mathbf{Z}, w)$, where $\hat{\psi}$ denotes here the Fourier transform of a periodic function of period $2 \pi$. Moreover $\psi$ may be chosen to be $\mathcal{C}^{\infty}$, if $\left|n^{r}\right| \leq K_{r} w(n)$ for all $n \in \mathbf{N}$, for all $r \in \mathbf{N}^{*}$, for some $K_{r}>0$.

Proof. The proof of 4.5 has to be done in several steps:

(a) First we define $t: \mathbf{R} \rightarrow \mathbf{R}$ by $t(x)=w([|x|])$ where $[z]$ denotes the integer part of $z$. Then the function $t$ satisfies the conditions stated in 2.2 with $k^{2}$ instead of $k$ and $l=l_{1} k$. In fact, if $x, y \geq 0$, let's put $x=[x]+a, y=[y]+b$ with $0 \leq a, b<1$. Hence $x+y=[x]+[y]+(a+b)$ with $0 \leq a+b<2$ and $[a+b]=0$ or 1 . Thus

$$
\begin{aligned}
t(x+y)=w([x+y]) \leq w([x] & +[y]+1) \\
& \leq C^{2} w^{k}(1) w^{k^{2}}([x]) w^{k^{2}}([y])=C_{1} t^{k^{2}}(x) t^{k^{2}}(y),
\end{aligned}
$$

where $C_{1}=C^{2} w^{k}(1)$. We may of course assume that $C_{1} \geq 1$. If $x, y \leq 0$,

$$
t(x+y)=t(-x-y) \leq C_{1} t^{k^{2}}(-x) t^{k^{2}}(-y)=C_{1} t^{k^{2}}(x) t^{k^{2}}(y) .
$$

If $x \geq 0, y \leq 0, x+y \geq 0$, then

$$
t(x+y)=w([x+y]) \leq w([x])=t(x) \leq C_{1} t^{k^{2}}(x) t^{k^{2}}(y) .
$$

The other cases are treated similarly. Moreover,

$$
\begin{gathered}
\int_{\mathbf{R}} t^{-2 l}(x) d x=2 \int_{0}^{+\infty} t^{-2 l}(x) d x=2 \int_{0}^{+\infty} w^{-2 l}([x]) d x=2 \sum_{n=0}^{+\infty} w^{-2 l}(n)<+\infty \\
\int_{\mathbf{R}} t^{-\left(l-k^{2}\right)}(x) d x=2 \int_{0}^{+\infty} w^{-\left(l-k^{2}\right)}([x]) d x=2 \sum_{n=0}^{+\infty} w^{-\left(l-k^{2}\right)}(n)<+\infty
\end{gathered}
$$


and

$$
\int_{\mathbf{R}} \frac{\ln t(x)}{1+x^{2}} d x=2 \sum_{n=0}^{+\infty} \int_{n}^{n+1} \frac{\ln w(n)}{1+x^{2}} d x \leq 2 \sum_{n=0}^{+\infty} \frac{\ln w(n)}{1+n^{2}}<+\infty .
$$

(b) Let now $\varphi$ be the function obtained in 4.3 and let's extend it to a periodic function of period $2 \pi$ called $\psi$. It remains to show that then

$$
\sum_{n \in \mathbf{Z}}|\hat{\psi}(n)| w(n)<+\infty
$$

Let's first evaluate, with the notations of 4.3 and because in this case $b-a \leq 2 \pi$,

$$
\begin{aligned}
|\hat{\psi}(n)| & =|\hat{\varphi}(n)| \\
& \leq\left|g_{1} * g_{2}(n)\right| \\
& \leq|g| *|\check{g}|(n) \\
& =\int_{\mathbf{R}} t^{-l}(n-y) t^{-l}(y) d y \\
& =\int_{\mathbf{R}} w^{-l}([|n-y|]) w^{-l}([|y|]) d y .
\end{aligned}
$$

For $n \in \mathbf{N}$,

$$
\begin{aligned}
|\hat{\psi}(n)| \leq & \int_{-\infty}^{0} w^{-l}([n-y]) w^{-l}([-y]) d y+\int_{0}^{n} w^{-l}([n-y]) w^{-l}([y]) d y \\
& +\int_{n}^{+\infty} w^{-l}([y-n]) w^{-l}([y]) d y \\
= & \sum_{m=-\infty}^{-1} w^{-l}(n-m-1) w^{-l}(-m-1)+\sum_{m=0}^{n-1} w^{-l}(n-m-1) w^{-l}(m) \\
& +\sum_{m=n}^{+\infty} w^{-l}(m-n) w^{-l}(m) \\
= & \sum_{m=-\infty}^{0} w^{-l}(n-m) w^{-l}(m)+\sum_{m=0}^{n-1} w^{-l}(n-m-1) w^{-l}(m) \\
& +\sum_{m=n}^{+\infty} w^{-l}(n-m) w^{-l}(m) .
\end{aligned}
$$

But

$$
w(n-m)=w(n-m-1+1) \leq C w^{k}(n-m-1) w^{k}(1)=C_{1} w^{k}(n-m-1)
$$


and

$$
w^{-l}(n-m-1) \leq C_{2} w^{-\frac{l}{k}}(n-m)
$$

for some positive constants $C_{1}$ and $C_{2}$. Finally, as $-l \leq-\frac{l}{k}=-l_{1}$,

$$
\begin{aligned}
|\hat{\psi}(n)| \leq & \sum_{m=-\infty}^{0} w^{-\frac{l}{k}}(n-m) w^{-\frac{l}{k}}(m)+C_{2} \sum_{m=0}^{n-1} w^{-\frac{l}{k}}(n-m) w^{-\frac{l}{k}}(m) \\
& +\sum_{m=n}^{+\infty} w^{-\frac{l}{k}}(n-m) w^{-\frac{l}{k}}(m) \\
\leq & C_{3} \sum_{m=-\infty}^{+\infty} w^{-l_{1}}(n-m) w^{-l_{1}}(m)
\end{aligned}
$$

for some new positive constant $C_{3}$. Similarly, for $n \in \mathbf{Z}_{-}$. This implies that

$$
\begin{aligned}
\sum_{n \in \mathbf{Z}}|\hat{\psi}(n)| w(n) & \leq C_{3} \sum_{n \in \mathbf{Z}}\left(\sum_{m \in \mathbf{Z}} w^{-l_{1}}(n-m) w^{-l_{1}}(m)\right) w(n-m+m) \\
& \leq C_{3} C \sum_{n \in \mathbf{Z}} \sum_{m \in \mathbf{Z}} w^{-l_{1}}(n-m) w^{k}(n-m) w^{-l_{1}}(m) w^{k}(m) \\
& =C_{3} C\left(\sum_{j \in \mathbf{Z}} w^{-\left(l_{1}-k\right)}(j)\right)^{2} \\
& <+\infty
\end{aligned}
$$

Remarks 4.6. (i) In order to get the existence of the function $\psi$ it is of course sufficient that the conditions of $w$ being increasing and $w(n+m) \leq C w^{k}(n) w^{k}(m)$ are satisfied for $n, m \geq N_{0}$ for some $N_{0} \in \mathbf{N}$. In fact, one may change the function $w$ to be constant for $|n| \leq N_{0}$. Then this new function satisfies the hypotheses for all $n \in \mathbf{N}$ and may be used to get $\psi$.

(ii) Assume for instance that $w(n) \geq C(1+|n|)^{\alpha}$, for some $\alpha>0$, for some $C>0$, for all $n$. Then the existence of $l, l_{1}$ such as in 4.4 is obvious.

The previous results are now used to construct functional calculus.

4.7. Let $f=f^{*}: G \rightarrow \mathbf{C}$ be continuous with compact support. Recall (3.12) that we have the following bound:

$$
\|u(n f)\|_{\omega} \leq K_{1}(1+|n|)\left(1+|n|^{2}\right)^{\frac{Q}{2}} s(|n|)^{2 \ln (\ln |n|)} e^{K_{3}\left(\frac{|n|}{(\ln |n|)^{C}}\right)}, \quad \text { with } C>1 .
$$

Let's call the right hand side of the previous inequality $w(n)$ for $|n| \geq e^{e}$ and let's see wether the hypotheses of 4.4 are satisfied. We have

$$
(1+n+m) \leq(1+n)(1+m) \leq(1+n)^{k}(1+m)^{k} \quad \forall k \geq 1, \forall n, m \in \mathbf{N}
$$


and

$$
\begin{aligned}
\left(1+(n+m)^{2}\right)^{\frac{Q}{2}} & \leq\left(\left(1+n^{2}\right)\left(1+m^{2}\right)+n m\right)^{\frac{Q}{2}} \\
& \leq 2^{\frac{Q}{2}}\left(1+n^{2}\right)^{\frac{Q}{2}}\left(1+m^{2}\right)^{\frac{Q}{2}} \\
& \leq 2^{\frac{Q}{2}}\left(\left(1+n^{2}\right)^{\frac{Q}{2}}\right)^{k}\left(\left(1+m^{2}\right)^{\frac{Q}{2}}\right)^{k} \quad \forall k \geq 1, \forall n, m \in \mathbf{N} .
\end{aligned}
$$

Moreover, we have

$$
\frac{n+m}{(\ln (n+m))^{C}} \leq \frac{n}{(\ln n)^{C}}+\frac{m}{(\ln m)^{C}}
$$

Hence,

$$
\begin{aligned}
e^{K_{3}\left(\frac{n+m}{(\ln (n+m))^{C}}\right)} & \leq e^{K_{3}\left(\frac{n}{(\ln (n))^{C}}\right)} e^{K_{3}\left(\frac{m}{(\ln (m))^{C}}\right)} \\
& \leq\left(e^{K_{3}\left(\frac{n}{(\ln (n))^{C}}\right)}\right)^{k}\left(e^{K_{3}\left(\frac{m}{(\ln (m))^{C}}\right)}\right)^{k}, \quad \forall k \geq 1, \forall n, m \geq e,
\end{aligned}
$$

and, if $n \geq m \geq e^{e}$,

$$
\begin{aligned}
\ln (\ln (n+m)) & =\ln \left(\ln \left(n\left(1+\frac{m}{n}\right)\right)\right) \\
& \leq \ln (\ln n+\ln 2) \\
& =\ln \left((\ln n)\left(1+\frac{\ln 2}{\ln n}\right)\right) \\
& \leq \ln (\ln n)+\ln 2 \\
& \leq \ln (\ln n)+\ln (\ln m)+\ln 2 \\
& \leq 2(\ln (\ln n)+\ln (\ln m)), \quad n, m \geq e^{e} .
\end{aligned}
$$

Similarly if $m \geq n \geq e^{e}$. Hence, if $n \geq m \geq e^{e}$,

$$
\begin{aligned}
s(n+m)^{2 \ln (\ln (n+m))} & \leq s(n)^{4 \ln (\ln (n+m))} \\
& \leq s(n)^{16 \ln (\ln n)} \\
& \leq s(n)^{16 \ln (\ln n)} s(m)^{16 \ln (\ln m)} \\
& =\left(s(n)^{2 \ln (\ln n)}\right)^{8}\left(s(m)^{2 \ln (\ln m)}\right)^{8} .
\end{aligned}
$$

Similarly for $m \geq n$. Hence the third condition of 4.4 is satisfied for $w$ and for $k=8$. As $w(n) \geq C(1+n)$, the fifth and sixth conditions of 4.4 are satisfied for the function 
$w$. The fourth condition of 4.4 admits the following equivalent formulations:

$$
\begin{aligned}
\sum_{n \in \mathbf{N}, n \geq e^{e}} & \frac{\ln (w(n))}{1+n^{2}}<+\infty \\
\Leftrightarrow & \sum_{n \in \mathbf{N}, n \geq e^{e}} \frac{\ln C_{1}}{1+n^{2}}+\sum_{n \in \mathbf{N}, n \geq e^{e}} \frac{\ln (1+n)}{1+n^{2}}+\sum_{n \in \mathbf{N}, n \geq e^{e}} \frac{Q \ln \left(1+n^{2}\right)}{2} \frac{n}{1+n^{2}} \\
& +2 \sum_{n \in \mathbf{N}, n \geq e^{e}} \frac{(\ln (\ln n)) \ln (s(n))}{1+n^{2}}+K_{2} \sum_{n \in \mathbf{N}, n \geq e^{e}} \frac{n}{(\ln n)^{C}\left(1+n^{2}\right)}<+\infty \\
\Leftrightarrow & \sum_{n \in \mathbf{N}, n \geq e^{e}} \frac{(\ln (\ln n)) \ln (s(n))}{1+n^{2}}<+\infty
\end{aligned}
$$

as the other sums converge for $C>1$.

This leads us to the main theorem:

Theorem 4.8. Let $G$ be a compactly generated locally compact group with polynomial growth. Let $U$ be a generating neighbourhood of $G$. For any weight $\omega$ on $G$, let's define $s(n)=\sup _{x \in U^{n}} \omega(x)$. Assume that

$$
\sum_{n \in \mathbf{N}, n \geq e^{e}} \frac{(\ln (\ln n)) \ln (s(n))}{1+n^{2}}<+\infty .
$$

Let $f=f^{*}: G \rightarrow \mathbf{C}$ continuous with compact support be arbitrary. Then, given any $p, q$, $\varepsilon$ such that $0<p<p+\varepsilon<q-\varepsilon<q<2 \pi$, there exists $\psi$ continuous of period $2 \pi$ such that $\operatorname{supp} \psi \cap[0,2 \pi] \subset[p, q], \psi \equiv 1$ on $[p+\varepsilon, q-\varepsilon]$ and

$$
\sum_{n \in \mathbf{Z}}\|u(n f)\|_{\omega}|\hat{\psi}(n)|<+\infty .
$$

Hence this defines a function

$$
\psi\{f\}=\sum_{n \in \mathbf{Z}} \hat{\psi}(n) u(n f) \in L^{1}(G, \omega)
$$

and the properties of functional calculus are satisfied. The condition (3) is independent of the choice of the generating neighbourhood $U$.

Proof. The only thing that remains to be proven is the independence of (3) of the choice of $U$. Let $U$ and $V$ be two generating neighbourhoods of $G$. Then there exist $p, q \in \mathbf{N}^{*}$ such that $U \subset V^{p}$ and $V \subset U^{q}$. Hence, if we write $s_{U}(n)=\sup _{x \in U^{n}} \omega(x)$ and $s_{V}(x)=\sup _{x \in V^{n}} \omega(x)$, then

$$
s_{U}(n) \leq s_{V}(n)^{p} \text { and } s_{V}(n) \leq s_{U}(n)^{q} .
$$


This implies that

$$
\begin{aligned}
\sum_{n \in \mathbf{N}, n \geq e^{e}} \frac{(\ln (\ln n)) \ln \left(s_{U}(n)\right)}{1+n^{2}} & \leq p \sum_{n \in \mathbf{N}, n \geq e^{e}} \frac{(\ln (\ln n)) \ln \left(s_{V}(n)\right)}{1+n^{2}} \\
& \leq p q \sum_{n \in \mathbf{N}, n \geq e^{e}} \frac{(\ln (\ln n)) \ln \left(s_{U}(n)\right)}{1+n^{2}}
\end{aligned}
$$

which proves the independence.

Definition 4.9. Under the hypotheses of the previous theorem, let's call the condition

$$
\sum_{n \in \mathbf{N}, n \geq e^{e}} \frac{(\ln (\ln n)) \ln (s(n))}{1+n^{2}}<+\infty
$$

the non-abelian Beurling-Domar condition and let's denote it (BDna). Hence (BDna) implies the existence of functional calculus in the weighted group algebra $L^{1}(G, \omega)$.

Examples 4.10. (i) Let $\omega(x) \leq e^{C|x|^{\alpha}}$ with $C>0,0 \leq \alpha<1$ be a sub-exponential weight. Then

$$
\sum_{n \in \mathbf{N}, n \geq e^{e}} \frac{C(\ln (\ln n)) n^{\alpha}}{1+n^{2}}<+\infty
$$

as $0 \leq \alpha<1$ and (BDna) is satisfied.

(ii) Let

$$
\omega(x)=e^{C \frac{|x|}{(\ln (1+|x|) \gamma}}
$$

with $C>0, \gamma>0$. Then (BDna) is equivalent to

$$
\sum_{n \in \mathbf{N}, n \geq e^{e}} C \frac{(\ln (\ln n)) \cdot n}{\left(1+n^{2}\right)(\ln (1+n))^{\gamma}}<+\infty \Leftrightarrow \sum_{n \in \mathbf{N}, n \geq e^{e}} \frac{\ln (\ln n)}{n(\ln n)^{\gamma}}<+\infty .
$$

This condition is satisfied exactly when $\gamma>1$.

(iii) If $\omega_{i}, i \in\{1, \ldots, n\}$ are a finite number of weights that satisfy all the condition (BDna), then the same is true for their product $\prod_{i=1}^{n} \omega_{i}$. For an infinite product of weights a supplementary convergence condition will of course be necessary as shown by the following examples.

(iv) Let

$$
\omega(x)=e^{\sum_{i \in \mathbf{N}} c_{i}|x|^{\gamma_{i}}}
$$


with $c_{i}>0, \sum_{i \in \mathbf{N}} c_{i}<+\infty, 0<\gamma_{i}<1, \gamma_{i} \uparrow 1$. Then (BDna) means

$$
\begin{aligned}
\sum_{n \in \mathbf{N}, n \geq e^{e}} \sum_{i \in \mathbf{N}} c_{i} \frac{(\ln (\ln n)) n^{\gamma_{i}}}{1+n^{2}}<+\infty & \Leftrightarrow \sum_{i \in \mathbf{N}} c_{i}\left(\sum_{n \in \mathbf{N}, n \geq e^{e}} \frac{\ln (\ln n)}{n^{2-\gamma_{i}}\left(1+n^{-2}\right)}\right)<+\infty \\
& \Leftrightarrow \sum_{i \in \mathbf{N}} c_{i} d_{i}<+\infty
\end{aligned}
$$

where, for all $i$,

$$
d_{i}=\sum_{n \in \mathbf{N}, n \geq e^{e}} \frac{\ln (\ln n)}{n^{2-\gamma_{i}}\left(1+n^{-2}\right)}<+\infty .
$$

Given the sequence $\left(\gamma_{i}\right)$, the numbers $d_{i}$ are determined, and (BDna) then gives the condition $\sum_{i \in \mathbf{N}} c_{i} d_{i}<+\infty$ on the growth of the coefficients $c_{i}$.

(v) Let

$$
\omega(x)=e^{\sum_{i \in \mathbf{N}} c_{i} \frac{|x|}{(\ln (1+|x|))^{\gamma i}}},
$$

where $c_{i}>0, \sum_{i \in \mathbf{N}} c_{i}<+\infty, 1<\gamma_{i}, \gamma_{i} \downarrow 1$. Then (BDna) means

$$
\begin{aligned}
\sum_{n \in \mathbf{N}, n \geq e^{e}} \sum_{i \in \mathbf{N}} c_{i} \frac{(\ln (\ln n)) \cdot n}{\left(1+n^{2}\right)(\ln (1+n))^{\gamma_{i}}}<+\infty & \\
\Leftrightarrow \sum_{i \in \mathbf{N}} c_{i} \sum_{n \in \mathbf{N}, n \geq e^{e}} \frac{\ln (\ln n)}{n(\ln (1+n))^{\gamma_{i}}\left(1+n^{-2}\right)} & <+\infty \\
& \Leftrightarrow \sum_{i \in \mathbf{N}} c_{i} e_{i}<+\infty,
\end{aligned}
$$

where, for all $i$,

$$
e_{i}=\sum_{n \in \mathbf{N}, n \geq e^{e}} \frac{\ln (\ln n)}{n(\ln (1+n))^{\gamma_{i}}\left(1+n^{-2}\right)}<+\infty .
$$

Given the sequence $\left(\gamma_{i}\right)$, the numbers $e_{i}$ are determined, and (BDna) then gives the condition $\sum_{i \in \mathbf{N}} c_{i} e_{i}<+\infty$ on the growth of the coefficients $c_{i}$.

(vi) Let

$$
\omega(x)=e^{C|x|}
$$

with $C>0$. Then

$$
\sum_{n \in \mathbf{N}, n \geq e^{e}} C \frac{(\ln (\ln n)) n}{1+n^{2}}
$$

diverges and (BDna) is not satisfied. 
Remarks 4.11. (i) Let's recall that for abelian groups $G$ a lot of work has been done by Beurling ([1], [2]) and Domar ([5]). See also [19]. They use the following condition to get harmonic harmonic analysis properties:

$$
\sum_{n \geq 1} \frac{\ln \omega\left(x^{n}\right)}{n^{2}}<+\infty, \quad \forall x \in G .
$$

In case $G=\mathbf{R}$ and $\omega$ increasing on $\mathbf{R}_{+}$, this is equivalent to

$$
\int_{\mathbf{R}} \frac{\ln \omega(s)}{1+s^{2}} d s<+\infty .
$$

It is then also equivalent to

$$
\sum_{n \geq 1} \frac{\ln s(n)}{1+n^{2}}<+\infty
$$

as, in that case, $s(n)=\omega(n)$, provided we take $U=[-1,1]$. This last condition may again be defined for an arbitrary locally compact, compactly generated group $G$ with polynomial growth and an arbitrary weight $\omega$ on $G$ : We then say that $\omega$ satisfies the abelian Beurling-Domar condition and we write (BDa) if

$$
\sum_{n \geq 1} \frac{\ln (s(n))}{1+n^{2}}<+\infty .
$$

Obviously (BDna) implies (BDa). But for rapidly growing weights, the presence of $\ln (\ln n)$ in (BDna) doesn't seem to affect the convergence very much. Hence for the weight

$$
\omega(x)=e^{C \frac{|x|}{(\ln (1+|x|))^{\gamma}}}
$$

both (BDna) and (BDa) are satisfied if and only if $\gamma>1$. So our result is almost the best possible result we may expect. For more comments on this question, see also the introduction and the chapter on the Wiener property.

(ii) Instead of using the result of Paley-Wiener in the construction of functional calculus, one may also use results of Mandelbrojt ([13], [14]).

\section{The algebra $L^{1}(G, \omega)$}

5.1. We first mention some properties of the Banach $*$-algebra $L^{1}(G, \omega)$ which hold for arbitrary locally compact groups and arbitrary weights $\omega$ and which are obtained by standard arguments. The left translations $a \mapsto{ }_{a} f$, where ${ }_{a} f(x)=f\left(a^{-1} x\right)$, are strongly continuous from $G$ to $L^{1}(G, \omega)$. The same is true for right translations. 
The algebra $L^{1}(G, \omega)$ admits bounded approximate identities. This property ensures that the closed left, right and two-sided ideals in $L^{1}(G, \omega)$ are just the closed left, right and two-sided translation invariant subspaces.

Let $\pi$ be a strongly continuous representation of $G$ on a Banach space such that for all $x \in G,\|\pi(x)\|_{o p} \leq C \cdot \omega(x)$ for some positive constant $C$. Then $\pi$ defines a representation of $L^{1}(G, \omega)$ by

$$
\pi^{\prime}(f)=\int_{G} f(x) \pi(x) d x .
$$

If the representation $\pi$ of $G$ is irreducible, then the same is true for the corresponding representation of $L^{1}(G, \omega)$. Conversely, let $\pi^{\prime}$ be a continuous representation of $L^{1}(G, \omega)$ on a Banach space $\mathcal{V}$. Suppose that $\pi^{\prime}$ is non-degenerate, i.e., that $\pi^{\prime}\left(L^{1}(G, \omega)\right) \mathcal{V}$ is dense in $\mathcal{V}$. Because of the existence of bounded approximate identities, the classical proof shows that there exists a representation $\pi$ of $G$ satisfying $\|\pi(x)\|_{o p} \leq C \cdot \omega(x)$ and such that

$$
\pi^{\prime}(f)=\int_{G} f(x) \pi(x) d x, \quad \text { for all } f \in L^{1}(G, \omega) .
$$

We are only interested in $*$-representations on Hilbert spaces. In this case we have:

If $\pi^{\prime}$ is a $*$-representation of $L^{1}(G, \omega)$ on a Hilbert space $\mathcal{H}$, then the corresponding representation $\pi$ of $G$ is unitary and $\pi^{\prime}$ is the restriction to $L^{1}(G, \omega)$ of a *representation of $L^{1}(G)$.

The previous remarks apply in particular to $\pi \in \hat{G}$ (the set of equivalence classes of topologically irreducible unitary representations of $G$ ). There is a bijection between $\hat{G}$ and the set of equivalence classes of topologically irreducible, continuous *-representations of $L^{1}(G, \omega)$.

For $\pi \in \hat{G}$, let's write ker $\pi \cap L^{1}(G, \omega)$ (resp. ker $\pi$ ) for the kernel of $\pi$ in $L^{1}(G, \omega)$ (resp. in $L^{1}(G)$ ). Let $\operatorname{Prim}_{*} L^{1}(G, \omega)$ (resp. $\operatorname{Prim}_{*} L^{1}(G)$ ) be the space of the kernels of the topologically irreducible $*$-representations of $L^{1}(G, \omega)$ (resp. $L^{1}(G)$ ). This space is endowed with the hull-kernel topology, i.e. the closed sets are of the form $h(k(A))$, where $A$ is an arbitrary subset of $\operatorname{Prim}_{*} L^{1}(G, \omega)$, where

$$
k(A)=\bigcap_{\operatorname{ker} \pi \cap L^{1}(G, \omega) \in A} \operatorname{ker} \pi \cap L^{1}(G, \omega)
$$

and where

$$
h(I)=\left\{\operatorname{ker} \pi \cap L^{1}(G, \omega) \mid I \subset \operatorname{ker} \pi \cap L^{1}(G, \omega)\right\} .
$$

Similarly for $\operatorname{Prim}_{*} L^{1}(G)$.

Let now $G$ be a compactly generated, locally compact group with polynomial growth and let $\omega$ be a weight on $G$ that satisfies (BDna). Hence functional calculus exists on a total part of $L^{1}(G, \omega)$. 
Notations. Let's note $\mathcal{C}_{c}(G)$ for the set of continuous functions with compact support on $G$. Let's denote by $\Phi$ the set of continuous functions $\varphi$ from $\mathbf{R}$ to $\mathbf{R}$, periodic of period $2 \pi$, with $\varphi(0)=0$, with $\operatorname{supp} \varphi$ compact, that operate by functional calculus on the set of the continuous, self-adjoint functions with compact support on $G$, given by 4.5 and 4.8 .

We may then prove the following proposition:

Proposition 5.2. Let $G$ and $\omega$ be as explained in 5.1. Let $C \subset \hat{G}, C_{1} \subset$ $\operatorname{Prim}_{*} L^{1}(G, \omega)$ and $C_{2} \subset \operatorname{Prim}_{*} L^{1}(G)$ given by $C_{1}=\left\{\operatorname{ker} \pi \cap L^{1}(G, \omega) \mid \pi \in C\right\}$, resp. $C_{2}=\{\operatorname{ker} \pi \mid \pi \in C\}$. Let $\rho \in \hat{G}$. Then the following are equivalent:

(i) $\operatorname{ker} \rho \cap L^{1}(G, \omega) \in{\overline{C_{1}}}^{\operatorname{Prim}_{*} L^{1}(G, \omega)}$

(ii) $\bigcap_{\pi \in C} \operatorname{ker} \pi \cap L^{1}(G, \omega) \subset \operatorname{ker} \rho \cap L^{1}(G, \omega)$

(iii) $\|\rho(f)\|_{o p} \leq \sup _{\pi \in C}\|\pi(f)\|_{o p} \quad \forall f \in \mathcal{C}_{c}(G)$

(iv) $\|\rho(f)\|_{o p} \leq \sup _{\pi \in C}\|\pi(f)\|_{o p} \quad \forall f \in L^{1}(G, \omega)$

(v) $\|\rho(f)\|_{o p} \leq \sup _{\pi \in C}\|\pi(f)\|_{o p} \quad \forall f \in L^{1}(G)$

(vi) $\bigcap_{\pi \in C} \operatorname{ker} \pi \subset \operatorname{ker} \rho$

(vii) $\operatorname{ker} \rho \in{\overline{C_{2}}}^{\operatorname{Prim}_{*}} L^{1}(G)$

(viii) $\rho \in \bar{C}^{\hat{G}}$

Proof. The equivalence of (vii) and (viii) is due to the fact that every locally compact group of polynomial growth is $*$-regular ([3]). The equivalences of (i) and (ii), resp. of (vi) and (vii), result from the definition of the hull-kernel topology. The implication (vi) $\Rightarrow$ (ii) is trivial. Recall moreover that $\mathcal{C}_{c}(G) \subset L^{1}(G, \omega) \subset L^{1}(G)$, that $\mathcal{C}_{c}(G)$ is dense in $L^{1}(G)$ and in $L^{1}(G, \omega)$ and that $\|\pi(f)\|_{o p} \leq\|f\|_{1}$ for every $f \in L^{1}(G)$. This proves the equivalence of (iii), (iv) and (v). The implication (v) $\Rightarrow$ (vi) is trivial. The last implication that has to be proven is (ii) $\Rightarrow$ (iii). Its proof uses standard arguments on functional calculus (see for instance [11]). Let's assume that (iii) is false. There exists $g \in \mathcal{C}_{c}(G)$ such that

$$
\sup _{\pi \in C}\|\pi(g)\|_{o p}<\|\rho(g)\|_{o p}
$$

We may of course assume that $g=g^{*}$ (by replacing $g$ by $g * g^{*}$ ) and that $\|g\|_{1} \leq 1$ (by dividing $g$ through $\|g\|_{1}$ ). Let's now take $\varphi \in \Phi$ such that $\varphi \equiv 0$ on a neighbourhood of $\left[-\sup _{\pi \in C}\|\pi(g)\|_{o p}, \sup _{\pi \in C}\|\pi(g)\|_{o p}\right]$ and such that $\varphi\left(\|\rho(g)\|_{o p}\right)=1$. Let $f=\varphi\{g\}$. Then, for every $\pi \in C, \pi(f)=\pi(\varphi\{g\})=\varphi(\pi(g))=0$ as $\varphi \equiv 0$ on the spectrum of $\pi(g)$, and $\rho(f)=\rho(\varphi\{g\})=\varphi(\rho(g)) \neq 0$ as $\varphi\left(\|\rho(g)\|_{o p}\right)=1$ and as $\|\rho(g)\|_{o p}$ is in the spectrum of $\rho(g)$. Hence $f \notin \operatorname{ker} \rho \cap L^{1}(G, \omega)$, but $f \in \bigcap_{\pi \in C} \operatorname{ker} \pi \cap L^{1}(G, \omega)$, which contradicts (ii). 
This proposition has several consequences:

Proposition 5.3. For all $\pi, \rho \in \hat{G}$,

$$
\operatorname{ker} \pi \cap L^{1}(G, \omega)=\operatorname{ker} \rho \cap L^{1}(G, \omega) \Longrightarrow \operatorname{ker} \pi=\operatorname{ker} \rho .
$$

If $G$ is a connected, simply connected, nilpotent Lie group, this implies moreover that $\pi \cong \rho$.

Proof. Take $C=\{\pi\}$ in 5.2 to show that $\operatorname{ker} \pi \cap L^{1}(G, \omega) \subset \operatorname{ker} \rho \cap L^{1}(G, \omega)$ implies $\operatorname{ker} \pi \subset \operatorname{ker} \rho$.

Proposition 5.4. The map

$$
\begin{aligned}
\Psi: \operatorname{Prim}_{*} L^{1}(G) & \longrightarrow \operatorname{Prim}_{*} L^{1}(G, \omega) \\
\operatorname{ker} \pi & \longmapsto \operatorname{ker} \pi \cap L^{1}(G, \omega)
\end{aligned}
$$

is a homeomorphism.

Proof. By 5.2 and 5.3.

Corollary 5.5. If $G$ is a connected, simply connected, nilpotent Lie group, then the sets $\left\{\operatorname{ker} \pi \cap L^{1}(G, \omega)\right\}$ are closed in $\operatorname{Prim}_{*} L^{1}(G, \omega)$, in particular

$$
\operatorname{ker} \pi \cap L^{1}(G, \omega) \subset \operatorname{ker} \rho \cap L^{1}(G, \omega) \Longrightarrow \pi \cong \rho \text {. }
$$

Proof. In this case, the same result is true for $L^{1}(G)$, as $G$ is a type I group. Apply 5.4 .

We then have Domar's property for the algebra $L^{1}(G, \omega)$ :

Theorem 5.6. Let $G$ be a locally compact, compactly generated group with polynomial growth. Let $\omega$ be a weight on $G$ that satisfies (BDna). Then, given any $\rho \in \hat{G}$ and any open neighbourhood $N$ of $\rho$, resp. given any open neighbourhood $N_{1}$ of $\operatorname{ker} \rho \cap L^{1}(G, \omega)$ in $\operatorname{Prim}_{*} L^{1}(G, \omega)$, there exists $f \in L^{1}(G, \omega)$ such that $\rho(f) \neq 0$ and $\pi(f)=0$ for all $\pi \in \hat{G} \backslash N$, resp. for all $\pi$ such that $\operatorname{ker} \pi \cap L^{1}(G, \omega) \in \operatorname{Prim}_{*} L^{1}(G, \omega) \backslash N_{1}$.

Proof. Put $C=\hat{G} \backslash N, C_{1}=\operatorname{Prim}_{*} L^{1}(G, \omega) \backslash N_{1}$ and apply 5.2.

The symmetry of the weighted group algebra is given by the following result:

Theorem 5.7. Let $G$ be a compactly generated, locally compact group with polynomial growth. Let $\omega$ be a weight on $G$. If $\omega$ satisfies condition $(\mathrm{S})$, then the algebra $L^{1}(G, \omega)$ is symmetric. This is in particular the case if $\omega$ satisfies (BDna). 
Proof. See [6] for the fact that $(S)$ implies the symmetry of the algebra. Let's show that (BDa), and hence (BDna), implies property $(S)$, i.e. the symmetry of the algebra. In fact, as the function $n \mapsto s(|n|)$ is a weight on $\mathbf{Z}, A=\lim _{n} \frac{\ln (s(n))}{n} \geq 0$ exists. Let's assume that $A>0$. Then

$$
\begin{aligned}
\sum_{n \geq 1} \frac{\ln (s(n))}{1+n^{2}} & =\sum_{n \geq 1} \frac{\ln (s(n))}{n} \cdot \frac{n}{1+n^{2}} \\
& \geq(A-\varepsilon) \sum_{n \geq N_{0}} \frac{1}{n\left(1+n^{-2}\right)}
\end{aligned}
$$

for some $\varepsilon>0$ such that $A-\varepsilon>0$ and for some $N_{0} \in \mathbf{N}$. As the last sum diverges, (BDa) cannot be satisfied.

The symmetry of the algebra and the previous results imply the following theorem:

Theorem 5.8. Let $G$ be a compactly generated, locally compact group with polynomial growth. Let $\omega$ be a weight on $G$ satisfying (BDna). Then the spaces Prim $G$, $\operatorname{Prim}_{*} L^{1}(G), \operatorname{Prim}_{*} L^{1}(G, \omega)$ are homeomorphic. Besides, the spaces $\operatorname{Prim} L^{1}(G)$ and $\operatorname{Prim} L^{1}(G, \omega)$ are homeomorphic to subspaces of $\operatorname{Prim}_{*} L^{1}(G)$, resp. $\operatorname{Prim}_{*} L^{1}(G, \omega)$.

\section{Minimal ideals}

6.1. In this chapter we are going to study the existence of minimal ideals of $L^{1}(G, \omega)$ of a given hull in $\operatorname{Prim}_{*} L^{1}(G, \omega)$. This study relies mainly on the work of [11]. Moreover it is connected to the problem of the Wiener property which we shall consider in Section 7. The hypotheses on the group $G$ and the weight $\omega$ will be the same as in 5 . For a given closed subset $C$ of $\operatorname{Prim}_{*} L^{1}(G, \omega)$ (identified with $\hat{G}$ ), let's introduce the following notations:

$$
\|f\|_{C}=\sup _{\pi \in C}\|\pi(f)\|_{o p}
$$

and

$$
\begin{aligned}
& m(C)=\left\{\varphi\{f\} \mid f=f^{*}, f \in \mathcal{C}_{c}(G),\|f\|_{1} \leq 1, \varphi \in \Phi,\right. \\
& \varphi\left.\equiv 0 \text { on a neighbourhood of }\left[-\|f\|_{C},\|f\|_{C}\right]\right\} .
\end{aligned}
$$

Let $j(C)$ be the closed two-sided ideal of $L^{1}(G, \omega)$ generated by $m(C)$. For $C=\emptyset$ we get

$$
\begin{aligned}
m(\emptyset)=\left\{\varphi\{f\} \mid f=f^{*}, f \in \mathcal{C}_{c}(G),\|f\|_{1} \leq 1, \varphi\right. & \in \Phi, \\
\varphi & \equiv 0 \text { on a neighbourhood of } 0\}
\end{aligned}
$$

An argument similar to the one in [11] gives the following result: 
Lemma 6.2. The hull of $j(C)$ is $C$.

Proof. If $C=\emptyset, C \subset h(j(C))$. Otherwise, take $\pi \in C$ and $\varphi\{f\} \in m(C)$. Then, $\|\pi(f)\|_{o p} \leq\|f\|_{C}$ and $\pi(\varphi\{f\})=\varphi(\pi(f))=0$, as $\varphi \equiv 0$ on the spectrum of $\pi(f)$. Hence $m(C) \subset \operatorname{ker} \pi$, $\operatorname{ker} \pi \in h(j(C))$ and $C \subset h(j(C))$.

Conversely, let $\rho \in \hat{G} \backslash C$. By 5.2 there exists $f \in \mathcal{C}_{c}(G)$ such that

$$
\|f\|_{C}=\sup _{\pi \in C}\|\pi(f)\|_{o p}<\|\rho(f)\|_{o p} .
$$

We may of course assume that $f=f^{*}$ (by replacing $f$ by $f * f^{*}$ ) and that $\|f\|_{1} \leq 1$ (by dividing by $\|f\|_{1}$ ). If $C=\emptyset$, replace $\|f\|_{C}$ by 0 . Hence there exists $\varphi \in \Phi$ such that $\varphi \equiv 0$ on a neighbourhood of $\left[-\|f\|_{C},\|f\|_{C}\right]$ and such that $\varphi\left(\|\rho(f)\|_{o p}\right) \neq 0$. By construction, $\varphi\{f\} \in m(C)$ and $\rho(\varphi\{f\})=\varphi(\rho(f)) \neq 0$ (as $\|\rho(f)\|_{o p}$ is in the spectrum of $\rho(f)$ and as $\left.\varphi\left(\|\rho(f)\|_{o p}\right) \neq 0\right)$. Hence $\operatorname{ker} \rho \notin h(j(C))$.

Because the algebra $L^{1}(G, \omega)$ is also symmetric, a result of Ludwig [11] gives us the existence of minimal ideals of a given hull, as stated in the following theorem:

Theorem 6.3. Let $G$ be a compactly generated, locally compact group with polynomial growth. Let $\omega$ be a weight on $G$ that satisfies condition (BDna). Let $C$ be a closed subset of $\hat{G}$. There exists a closed two-sided ideal $j(C)$ of $L^{1}(G, \omega)$, with $h(j(C))=C$, which is contained in every two-sided closed ideal $I$ with $h(I) \subset C$.

Proof. Take $\varphi\{f\} \in m(C)$ arbitrary. By 2.2 and 4.8 there exists $\psi \in \Phi$ such that $\varphi \cdot \psi=\psi \cdot \varphi=\varphi$. Hence $\psi\{f\} * \varphi\{f\}=\varphi\{f\}$ and $\psi\{f\} \in m(C)$. Moreover $h(\{\psi\{f\}\}) \supset h(m(C))=C$. We then apply lemma 2 of [11] to conclude.

\section{Wiener property}

Let us recall the following definition (see [6]):

Definition 7.1. Let $\mathcal{A}$ be a Banach *-algebra. We say that $\mathcal{A}$ has the Wiener property $(W)$ if for every proper closed two-sided ideal $I$ of $\mathcal{A}$, there exists a topologically irreducible $*$-representation $\pi$ of $\mathcal{A}$ such that $I \subset \operatorname{ker} \pi$. If $\mathcal{A}$ is of the form $L^{1}(G)$ for some locally compact group $G$, we also say that the group $G$ has the Wiener property.

Examples 7.2. (i) In [15] it is shown that if $G$ is a connected, simply connected, nilpotent Lie group and if $\omega$ is a polynomial weight on $G$, then $L^{1}(G, \omega)$ has the Wiener property.

(ii) In [6] it is shown that if $G$ is a compactly generated, locally compact group with polynomial growth and if $\omega$ is a weight on $G$ that is at most sub-exponential, then the algebra $L^{1}(G, \omega)$ has the Wiener property. 
(iii) For abelian groups, Domar ([5]) has shown that $L^{1}(G, \omega)$ has the Wiener property if $\sum_{n=1}^{+\infty} \frac{\ln \left(\omega\left(x^{n}\right)\right)}{n^{2}}<+\infty$ for all $x \in G$. This is in particular the case if $\sum_{n=1}^{+\infty} \frac{\ln (s(n))}{n^{2}}<+\infty$. For $G=\mathbf{R}$, Vretblad $([20])$ even shows the converse, for a certain type of weights: If $\omega(x)=\exp \left(\frac{\pi}{2}|x| q(x)\right)$ with $q$ decreasing on $\mathbf{R}_{+}$and $\omega$ increasing on $\mathbf{R}_{+}$and if $L^{1}(\mathbf{R}, \omega)$ has the Wiener property, then $\sum_{n=1}^{+\infty} \frac{\ln (\omega(n x))}{n^{2}}<+\infty$ (we write $n x$ instead of $x^{n}$ as $G=\mathbf{R}$ ) for every $x \in \mathbf{R}$ (see the introduction for more details).

In this section we shall study the Wiener property for algebras of the form $L^{1}(G, \omega)$, where $G$ is a compactly generated, locally compact group with polynomial growth and $\omega$ is a weight on $G$ satisfying condition (BDna), and hence such that $L^{1}(G, \omega)$ is a symmetric $*$-algebra that admits functional calculus. We shall first prove that in this situation the set $m(\emptyset)$ contains functions $\varphi\left\{f_{s}\right\}$ satisfying

$$
\left\|\varphi\left\{f_{s}\right\} * F-F\right\|_{\omega} \rightarrow 0
$$

in $L^{1}(G, \omega)$, for all $F \in \mathcal{C}_{c}(G)$. The techniques of the proof will be the same as those used in $([6])$ for the Wiener property, adapted to the new method of functional calculus.

7.3. Let $\left(f_{s}\right)_{s}$ be a bounded approximate identity in $L^{1}(G, \omega)$ such that, for all $s$,

$$
f_{s}=f_{s}^{*}, \quad\left\|f_{s}\right\|_{\omega} \leq C, \quad\left\|f_{s}\right\|_{1}=1, \quad \operatorname{supp} f_{s} \subset V_{s} \subset K,
$$

where $C$ is a positive constant, $V_{s}$ a compact symmetric neighbourhood of $e$ in $G$ and $K$ a fixed compact set. We shall show that there exists a periodic function $\varphi \in \Phi$ of period $2 \pi$ with $\varphi(1)=1, \varphi \equiv 0$ in a neighbourhood of 0 , such that

$$
\varphi\left\{f_{s}\right\}=\sum_{n \in \mathbf{Z}} \hat{\varphi}(n) u\left(n f_{s}\right)
$$

is converging for all $s$ and such that

$$
\left\|\varphi\left\{f_{s}\right\} * F-F\right\|_{\omega} \rightarrow 0
$$

for all continuous functions $F$ with compact support in $G$. Moreover the functions $\varphi\left\{f_{s}\right\}$ are contained in $m(\emptyset)$ by construction.

In fact, let's choose $\varphi \in \Phi$ such that $\varphi(1)=1$. Then

$$
\left\|\varphi\left\{f_{s}\right\} * F-F\right\|_{\omega}=\left\|\sum_{n \in \mathbf{Z}} \hat{\varphi}(n)\left[e^{i n f_{s}} * F-e^{i n} F\right]\right\|_{\omega} .
$$

As the functions $f_{s}$ are uniformly bounded in $L^{1}(G, \omega)$, it is easy to check that for every fixed $n$,

$$
e^{i n f_{s}} * F \rightarrow e^{i n} F
$$


in $L^{1}(G, \omega)$ as $s \rightarrow \infty$. So, for any fixed $N \in \mathbf{N}$, we have

$$
\sum_{|n| \leq N} \hat{\varphi}(n)\left[e^{i n f_{s}} * F-e^{i n} F\right] \rightarrow 0
$$

in $L^{1}(G, \omega)$. Next we have to show that we may choose $\varphi$ such that, for any $\varepsilon>0$, there exists $N \in \mathbf{N}$ such that

$$
\begin{aligned}
\left\|\sum_{|n|>N} \hat{\varphi}(n)\left[e^{i n f_{s}} * F-e^{i n} F\right]\right\|_{\omega} & \\
\leq & \sum_{|n|>N}|\hat{\varphi}(n)|\left\|e^{i n f_{s}} * F\right\|_{\omega}+\left|\sum_{|n|>N} \hat{\varphi}(n) e^{i n}\right|\|F\|_{\omega}<\varepsilon,
\end{aligned}
$$

independently of $s$. Suppose that we had already determined $\varphi$ and $N_{1}$ such that

$$
\sum_{|n|>N_{1}}|\hat{\varphi}(n)|\left\|e^{i n f_{s}} * F\right\|_{\omega}<\frac{\varepsilon}{2}
$$

for all $s$, then (as $\sum_{n \in \mathbf{Z}} \hat{\varphi}(n) e^{i n}=\varphi(1)=1$ converges) we can choose $N \geq N_{1}$ such that

$$
\left|\sum_{|n|>N} \hat{\varphi}(n) e^{i n}\right|\|F\|_{\omega}<\frac{\varepsilon}{2}
$$

Thus it suffices to show (4). According to 3.12

$$
\left\|e^{i n f_{s}} * F\right\|_{\omega} \leq K_{2}(1+|n|)\left(1+|n|^{2}\right)^{\frac{Q}{2}} s(|n|)^{2 \ln (\ln |n|)} e^{K_{3}\left(\frac{|n|}{(\ln |n|)}\right)}
$$

with constants $K_{2}$ and $K_{3}$ given by

$$
\begin{aligned}
& K_{2}=2^{\frac{Q}{2}}\left(K^{\frac{1}{2}}(1+q)^{\frac{Q}{2}} s(q)\|F\|_{2}+\|F\|_{\omega}\right) \\
& K_{3}=2 e^{\left(e^{C^{\prime} p}\right)\left\|f_{s}\right\|_{\omega}},
\end{aligned}
$$

where the constants $C, K, C^{\prime}$ just depend on the growth of the weight and where $q \in \mathbf{N}^{*}$ is such that $\operatorname{supp} F \subset U^{q}$. Moreover, as the support of the functions $f_{s}$ is contained in a fixed compact set, there exists $p \in \mathbf{N}^{*}$ such that $\operatorname{supp} f_{s} \subset U^{p}$ and hence $\left\|f_{s}\right\|_{\omega} \leq \sup _{x \in U^{p}} \omega(x)\left\|f_{s}\right\|_{1}=\sup _{x \in U^{p}} \omega(x)=s(p)$, for all $s$. Hence the constants $K_{2}$ and $K_{3}$ may in fact be chosen independently of $s$. Moreover, up to a constant, the bound is exactly the same as the one obtained for $\left\|u\left(n f_{s}\right)\right\|_{\omega}$. Hence, if the weight $\omega$ satisfies (BDna), there exist functions $\varphi \in \Phi$ satisfying the required conditions such that (4) holds. This proves the following result:

Theorem 7.4. Let $G$ be a compactly generated, locally compact group with polynomial growth. Let $\omega$ be a weight on $G$ that satisfies condition (BDna). Then $L^{1}(G, \omega)$ has the Wiener property. 
Proof. By 7.3, $j(\emptyset)$ contains all of $\mathcal{C}_{c}(G)$ and hence equals $L^{1}(G, \omega)$, as all the $\varphi\left\{f_{s}\right\}$ are in $m(\emptyset) \subset j(\emptyset)$. Finally, if $I$ is a closed two-sided ideal of $L^{1}(G, \omega)$ such that $h(I)=\emptyset$, then $L^{1}(G, \omega)=j(\emptyset) \subset I$ by 6.3 .

Examples 7.5. (i) In 4.10 (i), (iii) we have examples of weights that satisfy (BDna) and hence give a group algebra $L^{1}(G, \omega)$ that has the Wiener property.

(ii) Examples 4.10 (iv), (v) give certain growth conditions on the coefficients $c_{i}$ for the corresponding weight $\omega$ to satisfy (BDna). Under these conditions $L^{1}(G, \omega)$ has the Wiener property.

(iii) Let $\omega(x)=e^{C \frac{|x|}{(\ln (|x|+1))^{\gamma}}}$. Then $\omega$ satisfies (BDna) if and only if $\gamma>1$. Hence, for $\gamma>1, L^{1}(G, \omega)$ has the Wiener property. This is the best possible result. In fact, if $G=\mathbf{R}$, the result of Vretblad ([20]) shows that if $L^{1}(\mathbf{R}, \omega)$ has the Wiener property, then $\gamma>1$. So even if $G=\mathbf{R}$, we get the same condition on $\gamma$ that we had already in this paper for the non abelian case.

\section{References}

[1] A. Beurling, Sur les intégrales de Fourier absolument convergentes et leur application à une transformation fonctionnelle, 9ème Congrès des Mathématiciens Scandinaves, août 1938, Helsinki, 1939, pp. 345-366.

[2] _ Sur les spectres des fonctions, Analyse Harmonique., Colloques Internationaux du Centre National de la Recherche Scientifique, no. 15, Centre National de la Recherche Scientifique, Paris, 1949, pp. 9-29.

[3] J. Boidol, H. Leptin, J. Schürman, and D. Vahle, Räume primitiver Ideale von Gruppenalgebren, Math. Ann. 236 (1978), 1-13.

[4] J. Dixmier, Opérateurs de rang fini dans les représentations unitaires, Inst. Hautes Études Sci. Publ. Math. (1960), 13-25.

[5] Y. Domar, Harmonic analysis based on certain commutative Banach algebras, Acta Math. 96 (1956), 1-66.

[6] G. Fendler, K. Gröchenig, M. Leinert, J. Ludwig, and C. Molitor-Braun, Weighted group algebras on groups of polynomial growth, Math. Z. 245 (2003), 791-821.

[7] A. Hulanicki, Subalgebra of $L_{1}(G)$ associated with Laplacian on a Lie group, Colloq. Math. 31 (1974), 259-287.

[8] _ A functional calculus for Rockland operators on nilpotent Lie groups, Studia Math. 78 (1984), 253-266.

[9] J.-P. Kahane, Séries de Fourier absolument convergentes, Ergebnisse der Mathematik und ihrer Grenzgebiete, vol. 50, Springer-Verlag, Berlin, 1970.

[10] Y. Katznelson, An introduction to harmonic analysis, John Wiley \& Sons Inc., New York, 1968.

[11] J. Ludwig, Polynomial growth and ideals in group algebras, Manuscripta Math. 30 (1980), 215221.

[12] _ Minimal $C^{*}$-dense ideals and algebraically irreducible representations of the Schwartzalgebra of a nilpotent Lie group, Harmonic Analysis (Luxembourg, 1987), Lecture Notes in Math., vol. 1359, Springer, Berlin, 1988, pp. 209-217. 
[13] S. Mandelbrojt, Séries de Fourier et classes quasi-analytiques de fonctions, Gauthier-Villars, Paris, 1935.

[14] _ Séries adhérentes, régularisation des suites, applications, Gauthier-Villars, Paris, 1952.

[15] S. Mint Elhacen and C. Molitor-Braun, Étude de l'algèbre $L_{\omega}^{1}(G)$ avec $G$ groupe de Lie nilpotent et $\omega$ poids polynomial, Travaux mathématiques, Fasc. X, Sém. Math. Luxembourg, Centre Univ. Luxembourg, Luxembourg, 1998, pp. 77-94. (French, with English summary)

[16] R. E. A. C. Paley and N. Wiener, Fourier transforms in the complex domain, Colloquium Publications, vol. 19, AMS, New York, 1934.

[17] T. Pytlik, On the spectral radius of elements in group algebras, Bull. Acad. Polon. Sci. Sér. Sci. Math. Astronom. Phys. 21 (1973), 899-902. (English, with Russian summary)

[18] _ Symbolic calculus on weighted group algebras, Studia Math. 73 (1982), 169-176.

[19] H. Reiter, Classical harmonic analysis and locally compact groups, Clarendon Press, Oxford, 1968.

[20] A. Vretblad, Spectral analysis in weighted $L^{1}$ spaces on $\mathbf{R}$, Ark. Mat. 11 (1973), 109-138. 